\title{
ON THE EXISTENCE OF THE RATES OF STRESS AND DISPLACEMENT FOR PRANDTL-REUSS PLASTICITY*
}

BY

\section{GABRIELE ANZELLOTTI}

Libera Università degli Studi di Trento, Povo, Italy

Introduction. In this paper we shall consider an isotropic and homogeneous elasto-perfectly plastic body, subject to the Prandtl-Reuss constitutive law with the von Mises yield condition. We shall be concerned with the existence of the rate of stress and of the rate of displacement corresponding to a given state of stress, to a given rate of the force density, and to given boundary conditions.

We use the following notation. The body, in its nondeformed state, occupies a bounded connected open set $\Omega \subset \mathbb{R}^{3} ; \Gamma_{D}$ and $\Gamma_{N}$ are subsets of $\partial \Omega$ such that $\Gamma_{D} \cup \Gamma_{N}=\partial \Omega$, $\Gamma_{D} \cap \Gamma_{N}=\varnothing ; n(x)$ is the outward unit normal vector to $\partial \Omega$ at $x$. We are given a state of stress $\sigma=\left\{\sigma_{i j}\right\}_{i, j=1,2,3}: \Omega \rightarrow \mathbb{R}^{9}$ with $\sigma_{i j}=\sigma_{j i}$ and such that $\left|\sigma^{D}\right| \leqslant \sqrt{2} K$ on $\Omega$, where $\sigma^{D}$ is the deviator of $\sigma$ and $K$ is a positive constant called the yield constant. The functions $\dot{f}(x): \Omega \rightarrow \mathbb{R}^{3}, \dot{F}(x): \Gamma_{N} \rightarrow \mathbb{R}^{3}, \dot{g}(x): \Gamma_{D} \rightarrow \mathbb{R}^{3}$ are given.

We shall consider the following problem (see [7]):

Problem (P.1). Find under what conditions for the data there exists, in some space of functions or distributions, a triple $\{\dot{u}, \dot{\sigma}, \lambda\}$, where

$$
\begin{aligned}
& \dot{u}: \Omega \rightarrow \mathbb{R}^{3}, \\
& \dot{\boldsymbol{\sigma}}: \Omega \rightarrow \mathbb{R}^{9}, \quad \dot{\boldsymbol{\sigma}}=\left\{\dot{\boldsymbol{\sigma}}_{i j}\right\}_{i, j=1,2,3} \\
& \lambda: \Omega \rightarrow \mathbb{R},
\end{aligned}
$$

such that

$$
\begin{aligned}
\dot{\sigma}_{i j} & =\dot{\sigma}_{j i}, \\
\dot{\sigma}_{i j, j}+\dot{f}_{i} & =0 \text { in } \Omega, \\
\dot{\sigma}_{i j} n_{j} & =\dot{F}_{i} \text { on } \Gamma_{N}, \\
\varepsilon(\dot{u}) & =\frac{1}{9 K_{0}} \operatorname{trace} \dot{\boldsymbol{\sigma}} I+\frac{1}{2 \mu} \dot{\boldsymbol{\sigma}}^{D}+\lambda \sigma^{D},
\end{aligned}
$$

\footnotetext{
* Received February 15, 1982.
} 


$$
\begin{gathered}
\lambda \begin{cases}=0 & \text { where }\left|\sigma^{D}\right|<\sqrt{2} K, \text { or } \\
& \text { where }\left|\sigma^{D}\right|=\sqrt{2} K \text { and } \sigma^{D} \cdot \varepsilon^{D}(\dot{u})<0, \\
\geqslant 0 & \text { where }\left|\sigma^{D}\right|=\sqrt{2} K \text { and } \sigma^{D} \cdot \varepsilon^{D}(\dot{u}) \geqslant 0,\end{cases} \\
\dot{\sigma}(x) \cdot \sigma^{D}(x) \leqslant 0 \quad \text { if }\left|\sigma^{D}(x)\right|=\sqrt{2} K,
\end{gathered}
$$

where $\varepsilon_{i j}(\dot{u})=\frac{1}{2}\left(\partial \dot{u}_{i} / \partial x_{j}+\partial \dot{u}_{i} / \partial x_{i}\right)$.

We have used the summation convention over repeated indices. We denote by $\dot{\sigma}_{i j, j}$ the derivative $\left(\partial / \partial x_{j}\right) \dot{\sigma}_{i j}, K_{0}$ and $\mu$ are positive numbers, and $I$ is the identity matrix.

Notice that all the functions in problem (P.1) depend on the space variable $x$ only, and not on time. The dot on $\dot{u}$, $\dot{\sigma}$ does not mean differentiation with respect to time, and is there only to remind us that we are dealing with functions that satisfy the equation for the rates of the stresses and of the displacements.

In what follows, we shall consider problem (P.1) only in the case of Dirichlet boundary conditions (i.e. $\Gamma_{N}=\varnothing$ ) and in the case of Neumann boundary conditions (i.e. $\Gamma_{D}=\varnothing$ ). The case of mixed boundary conditions offers a few technical difficulties and we leave it to a subsequent paper.

The solution of problem (P.1) can be interesting from the point of view of a proof of the mathematical consistency of the theory of plasticity (Koiter [7, introduction]) and as a possible first step towards a solution of the evolution problem, particularly in the quasi-static case. A proof of the existence of weak solutions to these last problems is given in Duvaut and Lions [4, Chapter V] and in Suquet [10], but the point of view from which we are looking at the problem is different; it is close to that adopted in Temam [11] and Anzellotti and Giaquinta [1,2] for the existence of the stresses and displacements in the case of Hencky's total deformation theory. In fact, as in [2], we use the direct method of the calculus of variations to minimize a suitable energy functional in order to get the rate of displacement $\dot{u}$. Then we define $\dot{\sigma}$ and $\lambda$ starting from $\dot{u}$, and the constitutive law is satisfied automatically by $\dot{\sigma}, \lambda$ by the choice of the space $P_{\sigma}(\Omega)$ of the admissible displacement rates.

The basic notation and the definition of $P_{\sigma}(\Omega)$ are given in Sec. 1. We define the space $P_{\sigma}(\Omega)$ so that the deformation energy of a vector field $\dot{u} \in P_{\sigma}(\Omega)$ is finite; hence we say that $\dot{u} \in P_{\sigma}(\Omega)$ if:

(i) $\dot{u} \in \mathrm{BD}(\Omega)$, where $\mathrm{BD}(\Omega)$ is the space of functions of bounded deformation introduced and studied in $[8,9,5]$,

(ii) $\operatorname{div} \dot{u} \in L^{2}(\Omega)$,

(iii) in the elastic zone $A=\left\{x \in \Omega|| \sigma^{D}(x) \mid<\sqrt{2} K\right\}$, we have that $\varepsilon^{D}(\dot{u})$ is square summable,

(iv) in the elasto-plastic zone $C=\left\{x \in \Omega|| \sigma^{D}(x) \mid=\sqrt{2} K\right\}$, we have that $\varepsilon^{D}(\dot{u})$ is decomposed as the sum of an elastic part $e^{D}(\dot{u}) \in L^{2}(\Omega)$, such that $e^{D}(\dot{u}) \cdot \sigma^{D} \leqslant 0$, and a plastic part $\left(1 / 2 K^{2}\right)\left[\sigma^{D} \cdot \varepsilon^{D}(\dot{u})\right]^{+} \sigma^{D}$, which is in the direction of $\sigma^{D}$, where $\left[\sigma^{D} \cdot \varepsilon^{D}(\dot{u})\right]^{+}$ is a positive measure. 
In Sec. 2 we study the possible kind of discontinuity of a function $\dot{u} \in P_{\sigma}(\Omega)$ along a surface $\Gamma \subset \Omega$, and we prove that the difference $\left(\dot{u}^{+}-\dot{u}^{-}\right)$between the traces of $\dot{u}$ on the two sides of $\Gamma$ must be zero, not only at those points $x \in \Gamma$ where $\left|\sigma^{D}(x)\right|<\sqrt{2} K$, but at $\mathcal{H}^{2}$-almost all points $x$ where $\left|L_{t}(x)\right|<K$, where $L_{t}$ is the tangent part of the traction $L_{i}=\sigma_{i j} \nu_{j}(\nu$ is the normal to $\Gamma)$. Moreover, we prove that if the difference $\left(\dot{u}^{+}-\dot{u}^{-}\right)(x)$ is nonzero then it must be in the same direction as $L_{t}(x)$.

In Sec. 3 we give a meaning to the integrals $\int_{\partial \Omega} \sigma_{i j} u_{j} n_{i}$ and $\int_{\Omega} \sigma_{i j} \varepsilon_{i j}(u)$, for all $\sigma$ such that trace $\sigma \in L^{2}(\Omega),\left|\sigma^{D}\right|<+\infty, \sigma_{i j, j} \in L^{3}(\Omega)$, and for all $u \in \mathrm{BD}(\Omega)$ such that $\operatorname{div} u \in L^{2}(\Omega)$. These results follow mainly from an approximation theorem given in [2] and are needed for use in Sec. 4. For these results compare [6].

In Sec. 4 , we consider the functional

$$
E_{1}(\dot{u})=\frac{K_{0}}{2} \int_{\Omega}(\operatorname{div} \dot{u})^{2} d x+\mu \int_{\Omega}\left|e^{D}(\dot{u})\right|^{2}
$$

representing the energy of the elastic part of the deformation rate $\varepsilon(\dot{u})$. This energy does not take into account the plastic component of the deformation rate which is in the direction of $\sigma^{D}$; hence $E_{1}(\dot{u})$ cannot bound the integral $\int|\varepsilon(\dot{u})|^{2}$. However, it can be proved that, under essentially the safe load condition (H4.1) for the stress state $\sigma$, one has

$$
E_{1}(\dot{u}) \geqslant c \int_{\Omega}\left|\varepsilon^{D}(\dot{u})\right|-M
$$

for all $\dot{u} \in P_{\sigma}(\Omega)$. This fact gives the coerciveness of $E_{1}(\dot{u})$ on the space of all $\dot{u} \in P_{\sigma}(\Omega)$ that take, in a suitable sense, a given boundary value $\dot{g}$ (see Theorem 4.1). It is then proved (theorem 4.2) that the bounded sequences in $P_{\sigma}(\Omega)$ are compact with respect to a suitable $P_{\sigma}(\Omega)$-weak convergence (see Definition 4.2) and that the functional $E_{1}(\dot{u})$ is lower-semicontinuous with respect to this convergence. An existence theorem is finally given for the minimum problem:

$$
\begin{aligned}
E_{1}(\dot{u}) & =\min , \\
\mathscr{B}(\dot{g}-\dot{u}) & =0,
\end{aligned}
$$

where $\mathscr{B}(\dot{g}-\dot{u})=0$ is a set of boundary conditions that mean

$$
\begin{gathered}
(\dot{g}-\dot{u})(x)=0 \text { at points } x \in \partial \Omega \text { where the norm } \\
\text { of the tangent part } L_{t} \text { of the traction force } \\
\left\{\sigma_{i j} n_{j}\right\}_{i=1,2,3} \text { does not attain the yield } \\
\text { value } K ; \\
(\dot{g}-\dot{u})(x)=(1 / K)|\dot{g}-\dot{u}| L_{t} \text { if }(\dot{g}-\dot{u})(x) \neq 0 .
\end{gathered}
$$

In Sec. 5 , we show how one can obtain a solution $(\dot{u}, \dot{\sigma}, \lambda)$ to problem (P.1), in the case of Dirichlet boundary conditions, from every solution $\dot{u}$ to the minimum problem (P.2). As we have already said, we define $\dot{\sigma}$ and $\lambda$ starting from $\dot{u}$ (see (5.1)), and the constitutive laws are automatically satisfied, while the equilibrium conditions are checked using the Euler equation of the functional $E_{1}$.

The boundary condition $\dot{u}=\dot{g}$ is satisfied only in the weaker sense (0.6).

In Sec. 6 we study the problem of Neumann boundary condition. We introduce another 
functional $E_{2}(\dot{u})$ and we study a new minimum problem (P.3). We consider a space $\Sigma_{\sigma}(\Omega)$ of stress rates and we say that a stress rate $\dot{\alpha} \in \Sigma_{\sigma}(\Omega)$ is $(\dot{f}, \dot{F})$-admissible if

$$
\begin{aligned}
\dot{\alpha}_{i j \cdot j}+\dot{f}_{i} & =0 \quad \text { in } \Omega, \\
\alpha_{i j} n_{j} & =\dot{F}_{i} \quad \text { on } \Gamma_{N}
\end{aligned}
$$

hold (in a suitable weak sense). Clearly, in order to have a solution to our problem, we must impose conditions on $\dot{f}, \dot{F}$. We can prove that if $\dot{f}, \dot{F}$ are such that there exists a $(\dot{f}, \dot{F})$-admissible stress rate $\dot{\alpha}$, then the functional $E_{2}(\dot{u})$ is coercive; moreover, if $\dot{\alpha}$ can be found such that

$$
\dot{\alpha}_{i j}^{D} \cdot \sigma_{i j}^{D}=0 \text { in }\left\{x \in \Omega|| \sigma^{D}(x) \mid=\sqrt{2} K\right\}
$$

also holds, the functional $E_{2}(\dot{u})$ is lower-semicontinuous. Under these conditions we prove the existence of a solution to (P.3) and, as for the Dirichlet problem in Sec. 5, we find a solution $\{\dot{u}, \dot{\sigma}, \lambda\}$ to problem (P.1) (now in the case of $\Gamma_{D}=\varnothing$ ).

It must be noticed that the existence of a $(\dot{f}, \dot{F})$-admissible stress rate is a necessary condition in order to have a solution to the minimum problem (P.3); and condition (0.7), too, must be satisfied by $\dot{\alpha}(x, t)=(\partial / \partial t) \sigma(x, t)$ if $\sigma$ is a solution to the quasi-static problem and is differentiable with respect to the time $t$. On the other hand, condition (0.7) seems unnecessary for the existence of $\dot{u}, \dot{\sigma}$ in our case, and it would be desirable to have a proof of the semicontinuity of $E_{2}(\dot{u})$ that does not rely on (0.7).

Finally, at the end of Sec. 6, it is proved (see Theorem 6.4) that the stress rate $\dot{\sigma}$, in the case of Neumann boundary conditions, minimizes a complementary energy functional among the $(\dot{f}, \dot{F})$-admissible stress rates. Since this complementary energy is a strictly convex functional, we obtain that the stress rate $\dot{\sigma}$ is unique.

I would like to thank Mariano Giaquinta for many stimulating conversations, and G. Strang, R. Temam, and P. Villaggio for some useful comments.

1. In this section we set notation and define a space $P_{\sigma}(\Omega)$ of vector fields in $\Omega$. This is the space in which we shall later look for $\dot{u}$.

We shall assume that $\Omega$ is a bounded connected open set in $\mathbb{R}^{3}$ with a Lipschitz boundary. Recall that a vector field $u: \Omega \rightarrow \mathbb{R}^{3}$ is said to be of bounded deformation $(u \in \mathrm{BD}(\Omega))$ if $u \in L^{1}(\Omega)$ and the distributions

$$
\varepsilon_{i j}(u)=\frac{1}{2}\left(\frac{\partial u_{i}}{\partial x_{j}}+\frac{\partial u_{i}}{\partial x_{i}}\right)
$$

are measures of bounded variation in $\Omega$. The space $\operatorname{BD}(\Omega)$ is given the norm

$$
\|u\|_{\mathrm{BD}(\Omega)}=\int_{\Omega}|u| d x+\int_{\Omega}|\varepsilon(u)|
$$

where, for all Borel sets $B \subset \Omega, \int_{B}|\varepsilon(u)|$ denotes the value on $B$ of the measure total variation associated to the vector measure $\varepsilon(u)=\left\{\varepsilon_{i j}(u)\right\}_{i, j=1,2,3}$.

For the properties of the space $\operatorname{BD}(\Omega)$ we refer to $[9,5]$ and also to $[1,2]$. For the convenience of the reader, we collect here a few results that will be needed for what follows. 
TheOREM 1.1 (a Sobolev-Poincaré inequality). Let $g=\left\{a \wedge x+b \mid a, b \in \mathbb{R}^{3}\right\}$ be the space of infinitesimal rigid motions in $\mathbb{R}^{3}$. For any continuous linear functional $\mathrm{T}: \mathrm{BD}(\Omega)$ $\rightarrow \mathscr{q}$ such that $\mathrm{T}(\eta)=\eta$ for all $\eta \in \mathscr{G}$, there exists a constant $c(\Omega, T)$ such that

$$
\|u-T(u)\|_{L^{3 / 2}(\Omega)} \leqslant c(\Omega, T) \int_{\Omega}|\varepsilon(u)|
$$

for all $u \in \mathrm{BD}(\Omega)$.

THEOREM 1.2 (a Poincaré inequality with compact support). There exists a constant $c(\Omega)$ such that

$$
\|u\|_{L^{\prime}(\Omega)} \leqslant c(\Omega)\left\{\int_{\Omega}|\varepsilon(u)|+\int_{\partial \Omega}|u| d \mathcal{H}^{2}\right\}
$$

for all $u \in \mathrm{BD}(\Omega)$.

THEOREM 3.1 (compactness). For any sequence of functions $u_{h} \in \mathrm{BD}(\Omega)$ such that

$$
\left\|u_{h}\right\|_{\mathrm{BD}(\Omega)} \leqslant M<+\infty \text { for all } h
$$

there exists a subsequence that converges in $L^{1}(\Omega)$ to some $u \in \mathrm{BD}(\Omega)$.

THEOREM 1.4 (trace theorem). There exists a linear operator $\gamma: \mathrm{BD}(\Omega) \rightarrow L^{1}(\partial \Omega)$ such that $\gamma(u)=\left.u\right|_{\partial \Omega}$ for all $u \in \operatorname{BD}(\Omega) \cap C^{0}(\bar{\Omega})$ and such that

$$
\int_{\partial \Omega}|\gamma(u)| d \mathcal{H}^{2} \leqslant c(\Omega)\|\varepsilon(u)\|_{\mathrm{BD}(\Omega)} \quad \text { for all } u \in \mathrm{BD}(\Omega) .
$$

Moreover, the following Green's formula holds for all $\phi \in C^{1}(\bar{\Omega})$ :

$$
\int_{\Omega} \varphi \varepsilon_{i j}(u)+\frac{1}{2} \int_{\Omega}\left[u_{i} \frac{\partial \varphi}{\partial x_{j}}+u_{j} \frac{\partial \varphi}{\partial x_{i}}\right] d x=\frac{1}{2} \int_{\partial \Omega} \varphi\left[n_{i} \gamma(u)_{j}+n_{j} \gamma(u)_{i}\right] d \mathcal{K}^{2} .
$$

For the sake of brevity, we shall usually write $u$ instead of $\gamma(u)$.

We shall make the following assumptions on the stress state $\sigma$ :

$$
\begin{aligned}
& \text { the deviator } \sigma^{D} \text { of } \sigma \text { is continuous in } \Omega, \\
& \left|\sigma^{D}(x)\right| \leqslant \sqrt{2} K \text { for all } x \in \Omega,
\end{aligned}
$$

trace $\sigma \in L^{2}(\Omega)$.

For all $\dot{u} \in \mathrm{BD}(\Omega)$ we consider the real-valued measure $\sigma^{D} \cdot \varepsilon^{D}(\dot{u})$, defined in $\Omega$ by

$$
\left\langle\sigma^{D} \cdot \varepsilon^{D}(\dot{u}), \varphi\right\rangle=\left\langle\varepsilon_{i j}^{D}(\dot{u}), \varphi \sigma_{i j}^{D}\right\rangle \text { for all } \varphi \in C_{0}^{0}(\Omega) \text {. }
$$

The elastic and plastic zones are

$$
A=\left\{x \in \Omega|| \sigma^{D}(x) \mid<\sqrt{2} K\right\}, \quad C=\left\{x \in \Omega|| \sigma^{D}(x) \mid=\sqrt{2} K\right\} .
$$

Definition 1.1. (i) We denote by $\left[\sigma^{D} \cdot \varepsilon^{D}(\dot{u})\right]$ the "restriction to $C$ " of the measure $\sigma^{D} \cdot \varepsilon^{D}(\dot{u})$. More precisely, $\left[\sigma^{D} \cdot \varepsilon^{D}(\dot{u})\right]$ is a measure on $\Omega$ such that

$$
\int_{B}\left[\sigma^{D} \cdot \varepsilon^{D}(\dot{u})\right]=\int_{B \cap C} \sigma^{D} \cdot \varepsilon^{D}(\dot{u})
$$

for all Borel subsets $B$ of $\Omega$. 
(ii) We define a vector valued measure $\varepsilon^{D}(\dot{u})_{\perp}$ in $\Omega$ as

$$
\varepsilon^{D}(\dot{u})_{\perp}=\varepsilon^{D}(\dot{u})-\left[\sigma^{D} \cdot \varepsilon^{D}(\dot{u})\right] \frac{\sigma^{D}}{\left|\sigma^{D}\right|^{2}}=\varepsilon^{D}(\dot{u})-\frac{1}{2 K^{2}}\left[\sigma^{D} \cdot \varepsilon^{D}(\dot{u})\right] \sigma^{D}
$$

where $\left\langle\left[\sigma^{D} \cdot \varepsilon^{D}(\dot{u})\right] \sigma^{D}, \varphi\right\rangle=\left\langle\left[\sigma^{D} \cdot \varepsilon^{D}(\dot{u})\right], \sigma^{D} \varphi\right\rangle$ for all $\varphi \in C_{0}^{0}\left(\Omega, \mathbb{R}^{9}\right)$.

(iii) We denote by $\left[\sigma^{D} \cdot \varepsilon^{D}(\dot{u})\right]^{-}$and $\left[\sigma^{D} \cdot \varepsilon^{D}(\dot{u})\right]^{+}$the negative and the positive part of the measure $\left[\sigma^{D} \cdot \varepsilon^{D}(\dot{u})\right]$.

Definition 1.2. (i) We shall say that the measure $\varepsilon^{D}(\dot{u})_{\perp}$ is an $L^{2}$-function in $\Omega$ if there exist functions $\gamma_{i j} \in L^{2}(\Omega)$ such that

$$
\left\langle\varepsilon^{D}(\dot{u})_{\perp}, \varphi\right\rangle=\int_{\Omega} \gamma_{i j}(x) \varphi_{i j}(x) d x
$$

for all $\varphi=\left\{\varphi_{i j}\right\} \in C_{0}^{0}\left(\Omega, \mathbb{R}^{9}\right)$. In such a case we use also the symbol $\varepsilon_{i j}^{D}(\dot{u})_{\perp}$ to denote the functions $\gamma_{i j}(x)$.

(ii) We shall says that the measure $\left[\sigma^{D} \cdot \varepsilon^{D}(u)\right]^{-}$is an $L^{2}$-function in $\Omega$ if there exists a function $\eta \in L^{2}(\Omega)$ such that

$$
\left\langle\left[\sigma^{D} \cdot \varepsilon^{D}(\dot{u})\right]^{-}, \varphi\right\rangle=\int_{\Omega} \eta(x) \varphi(x) d x
$$

for all $\varphi \in C_{0}^{0}(\Omega)$ (we must have $\eta(x)=0$ almost everywhere in $A$ ).

Definition 1.3. Set

$$
P_{\sigma}(\Omega)=\left\{\dot{u} \in \mathrm{BD}(\Omega) \mid \operatorname{div} \dot{u} \in L^{2}(\Omega), \varepsilon^{D}(\dot{u})_{\perp} \in L^{2}(\Omega),\left[\sigma^{D} \cdot \varepsilon^{D}(\dot{u})\right]^{-} \in L^{2}(\Omega)\right\} .
$$

Notice that if $\dot{u} \in P_{\mathrm{o}}(\Omega)$ one has in particular that $\varepsilon(\dot{u}) \in L^{2}(A)$.

Definition 1.4. For all functions $\dot{u} \in \mathrm{BD}(\Omega)$ set

$$
e^{D}(\dot{u})=\varepsilon^{D}(\dot{u})_{\perp}-\left(1 / 2 K^{2}\right)\left[\sigma^{D} \cdot \varepsilon^{D}(\dot{u})\right]^{-} \sigma^{D} .
$$

Notice that

$$
\varepsilon^{D}(\dot{u})=e^{D}(\dot{u})+\left(1 / 2 K^{2}\right)\left[\sigma^{D} \cdot \varepsilon^{D}(\dot{u})\right]^{+} \sigma^{D}
$$

and that

$$
e^{D}(\dot{u})=\varepsilon^{D}(\dot{u}) \quad \text { in } A .
$$

On the space $P_{\sigma}(\Omega)$ we shall take the norm

$$
\|\dot{u}\|_{P_{o}(\Omega)}=\|\dot{u}\|_{L^{1}(\Omega)}+\int_{\Omega}\left|\varepsilon^{D}(\dot{u})\right|+\|\operatorname{div} \dot{u}\|_{L^{2}(\Omega)}+\left\|e^{D}(\dot{u})\right\|_{L^{2}(\Omega)} .
$$

Remark 1.1. The space $P_{\sigma}(\Omega)$ has been defined so that if $\dot{u} \in P_{\sigma}(\Omega)$, then $\varepsilon(\dot{u})$ is square summable in the elastic zone $A$, while in the plastic zone $C, \varepsilon(\dot{u})$ is decomposed as the sum of an elastic part $e^{D}(\dot{u}) \in L^{2}(\Omega)$ such that $\sigma^{D} \cdot e^{D}(\dot{u}) \leqslant 0$ and a plastic part $\left(1 / 2 K^{2}\right)\left[\sigma^{D} \cdot \varepsilon^{D}(\dot{u})\right]^{+} \sigma^{D}$ in the direction of $\sigma^{D}$.

2. It is well known that functions of bounded deformation may have discontinuities along a surface; in this section we shall show that, with the additional requirement $\operatorname{div} \dot{u} \in L^{2}(\Omega), e^{D}(\dot{u}) \in L^{2}(\Omega)$, the discontinuity of $\dot{u}$ must be of a particular type. 
Let $V_{1}$ be an open set in $\mathbb{R}^{3}$ such that $V_{1} \cap \partial \Omega$ is non-empty, and set $\Gamma=V_{1} \cap \partial \Omega$, $\Omega_{1}=\Omega \cup V_{1}$. For all $\dot{u} \in \mathrm{BD}\left(\Omega_{1}\right)$ set

$$
\dot{u}^{+}=\text {trace of }\left.\dot{u}\right|_{\Omega_{1} \backslash \Omega} \text { on } \Gamma, \quad \dot{u}^{-}=\text {trace of }\left.\dot{u}\right|_{\Omega} \text { on } \Gamma \text {. }
$$

For all functions $v: \Gamma \rightarrow \mathbb{R}^{3}$ set, as usual,

$$
\tau_{i j}(v)=\frac{1}{2}\left[v_{i} n_{j}+v_{j} n_{j}\right], \quad n \text { is the normal to } \partial \Omega .
$$

Recall that the measure $\left.\varepsilon(\dot{u})\right|_{\Gamma}$ can be represented by integration on $\Gamma$ [1] as follows, where $\mathcal{H}^{2}$ is the two-dimensional Hausdorff measure and $B$ is any Borel subset of $\Gamma$ :

$$
\begin{aligned}
& \int_{B} \varepsilon(\dot{u})=\int_{B} \tau\left(\dot{u}^{+}-\dot{u}^{-}\right) d \mathcal{H}^{2}, \\
& \int_{B} \varepsilon^{D}(\dot{u})=\int_{B} \tau^{D}\left(\dot{u}^{+}-\dot{u}^{-}\right) d \mathcal{H}^{2}, \\
& \int_{B} \operatorname{trace} \varepsilon(\dot{u})=\int_{B} \operatorname{div} \dot{u}=\int_{B}\left(\dot{u}^{+}-\dot{u}^{-}\right) \cdot n d \mathcal{H}^{2} \\
&=\int_{B} \operatorname{trace}\left(\tau\left(\dot{u}^{+}-\dot{u}^{-}\right)\right) d \mathcal{H}^{2}, \\
& \int_{B}|\varepsilon(\dot{u})|=\int_{B}\left|\tau\left(\dot{u}^{+}-\dot{u}^{-}\right)\right| d \mathcal{H}^{2} .
\end{aligned}
$$

Moreover, when $\left(\dot{u}^{+}-\dot{u}^{-}\right) \cdot n=0 \mathcal{H}^{2}$-almost everywhere on $\Gamma$, one has

$$
\int_{B}|\varepsilon(\dot{u})|=\int_{B}\left|\varepsilon^{D}(\dot{u})\right|=\frac{1}{\sqrt{2}} \int_{B}\left|\dot{u}^{+}-\dot{u}^{-}\right| d \mathcal{H}^{2} .
$$

Now, assume that $\sigma$ is a stress field in $\Omega_{1}$ satisfying the hypotheses (H.1.1) in $\Omega_{1}$, and set

$$
\begin{gathered}
C_{1}=\left\{x \in \Omega_{1}|| \sigma^{D}(x) \mid=\sqrt{2} K\right\}, \quad A_{1}=\left\{x \in \Omega_{1}|| \sigma^{D}(x) \mid<\sqrt{2} K\right\}, \\
L=\left(L_{1}, L_{2}, L_{3}\right), \quad L_{i}(x)=\sigma_{i j}^{D}(x) n_{j}(x) \text { for } i=1,2,3, \\
{\left[\left(\dot{u}^{+}-\dot{u}^{-}\right) \cdot L\right]^{+}=\max \left\{\left(\dot{u}^{+}-\dot{u}^{-}\right) \cdot L, 0\right\},\left[\left(\dot{u}^{-}-\dot{u}^{-}\right) \cdot L\right]^{-}=-\min \left\{\left(\dot{u}^{+}-\dot{u}^{-}\right) \cdot L, 0\right\} .}
\end{gathered}
$$

We have the following

Proposition 2.1. For all $\dot{u} \in \mathrm{BD}\left(\Omega_{1}\right)$ and for all Borel subsets $B$ of $\Gamma$ we have

$$
\int_{B}\left[\sigma^{D} \cdot \varepsilon^{D}(\dot{u})\right]=\int_{B \cap C_{1}} \sigma^{D} \cdot \tau^{D}\left(\dot{u}^{+}-\dot{u}^{-}\right) d \mathcal{H}^{2}
$$

Moreover, if $\left(\dot{u}^{+}-\dot{u}^{-}\right) \cdot n=0, \mathcal{H}^{2}$-almost everywhere on $\Gamma$, we have

$$
\begin{gathered}
\int_{B}\left[\sigma^{D} \cdot \varepsilon^{D}(\dot{u})\right]=\int_{B \cap C_{1}}\left(\dot{u}^{+}-\dot{u}^{-}\right) \cdot L d \mathcal{H}^{2}, \\
\int_{B}\left[\sigma^{D} \cdot \varepsilon^{D}(\dot{u})\right]^{+}=\int_{B \cap C_{1}}\left[\left(\dot{u}^{+}-\dot{u}^{-}\right) \cdot L\right]^{+} d \mathcal{H}^{2},
\end{gathered}
$$




$$
\begin{gathered}
\int_{B}\left[\sigma^{D} \cdot \varepsilon^{D}(\dot{u})\right]^{-}=\int_{B \cap C_{1}}\left[\left(\dot{u}^{+}-\dot{u}^{-}\right) \cdot L\right]^{-} d \mathcal{H}^{2}, \\
\int_{B} \varepsilon^{D}(\dot{u})_{\perp}=\int_{B} \tau^{D}\left(\dot{u}^{+}-\dot{u}^{-}\right) d \mathcal{H}^{2}-\int_{B \cap C_{1}}\left[\left(\dot{u}^{+}-\dot{u}^{-}\right) \cdot L\right] \sigma^{D} d \mathcal{H}^{2},
\end{gathered}
$$

Proof. By definition 1.1, we have, for all Borel subsets $B$ of $\Gamma$,

$$
\int_{B}\left[\sigma^{D} \cdot \varepsilon^{D}(\dot{u})\right]=\int_{B \cap C_{1}} \sigma^{D} \cdot \varepsilon^{D}(\dot{u})
$$

and, as a consequence of the representation formula (2.2), we have

$$
\int_{B \cap C_{1}} \sigma^{D} \cdot \varepsilon^{D}(\dot{u})=\int_{B \cap C_{1}} \sigma^{D} \cdot \tau^{D}\left(\dot{u}^{+}-\dot{u}^{-}\right) d \mathcal{H}^{2}
$$

hence (2.6) follows.

Now, recall that if $\left(\dot{u}^{+}-\dot{u}^{-}\right) \cdot n=0$ one has $\tau\left(\dot{u}^{+}-\dot{u}^{-}\right)=\tau^{D}\left(\dot{u}^{+}-\dot{u}^{-}\right)$so that, also taking into account the symmetry of $\sigma$, one gets

$$
\tau_{i j}^{D}\left(\dot{u}^{+}-\dot{u}^{-}\right) \sigma_{i j}^{D}=\left(\dot{u}^{+}-\dot{u}^{-}\right){ }_{i} L_{i}
$$

and (2.7) follows.

Formula (2.7) says that the function $\left.\left[\left(\dot{u}^{+}-\dot{u}^{-}\right) \cdot L\right]\right|_{C_{1}}$ is the density of the measure $\left[\sigma^{D} \cdot \varepsilon^{D}(\dot{u})\right]$, with respect to the measure $\mathcal{H}^{2}$, on $\Gamma$ and formulae $(2.8)$ are then just a corollary of the Radon-Nikodym theorem.

Finally, (2.9) follows from the definition of $\varepsilon^{D}(\dot{u})_{\perp}$ and from (2.2) and (2.7). Q.E.D.

In the following two theorems we shall prove that, for a function $\dot{u} \in P_{\sigma}\left(\Omega_{1}\right)$, the difference $\left(\dot{u}^{+}-\dot{u}^{-}\right)$must be in the same direction as the vector $L_{t}=L-(L \cdot n) n$.

THEOREM 2.1. For all $\dot{u} \in P_{\sigma}\left(\Omega_{1}\right)$ we have that

$$
\begin{gathered}
\dot{u}^{+}-\dot{u}^{-}=0 \quad \mathcal{H}^{2} \text {-a.e. in }\left\{x \in \Gamma|| \sigma^{D}(x) \mid<\sqrt{2} K\right\}, \\
\left(\dot{u}^{+}-\dot{u}^{-}\right) \cdot n=0 \quad \mathcal{H}^{2} \text {-a.e. in } \Gamma, \\
{\left[\left(\dot{u}^{+}-\dot{u}^{-}\right) \cdot L\right]^{-}=0 \quad \mathcal{H}^{2} \text {-a.e. in } \Gamma,} \\
\tau^{D}\left(\dot{u}^{+}-\dot{u}^{-}\right)-\left[\left(\dot{u}^{+}-\dot{u}^{-}\right) \cdot L\right] \sigma^{D}=0 \quad \mathcal{K}^{2} \text {-a.e. in } \Gamma, \\
\int_{B}|\varepsilon(\dot{u})|=\frac{1}{\sqrt{2} K} \int_{B \cap C_{1}}\left[\left(\dot{u}^{+}-\dot{u}^{-}\right) \cdot L\right]^{+} \quad \text { for all Borel sets } B \subset \Gamma, \\
\left|\dot{u}^{+}-\dot{u}^{-}\right|=\frac{1}{K}\left(\dot{u}^{+}-\dot{u}^{-}\right) \cdot L \quad \mathcal{H}^{2} \text {-a.e. in } \Gamma .
\end{gathered}
$$

Proof. Recall that if $\dot{u} \in P_{\sigma}\left(\Omega_{1}\right)$ one has $e^{D}(\dot{u}) \in L^{2}\left(\Omega_{1}\right)$; hence $\varepsilon^{D}(\dot{u}) \in L^{2}\left(A_{1}\right)$ and also $\varepsilon(\dot{u}) \in L^{2}\left(A_{1}\right)$, so that $\int_{B} \varepsilon(\dot{u})=0$ for all sets $B \subset A_{1}$ of Lebesgue measure zero; this holds in particular for all sets $B \subset \Gamma \cap A_{1}$ and (2.10) follows then from (2.1).

To prove (2.11), (2.12) and (2.13) one can follow the same method. As an example we shall prove (2.12) (for that, we assume 2.11 has already been proved, so that (2.6) holds): since $\dot{u} \in P_{\sigma}\left(\Omega_{1}\right)$, we have that the measure $\left[\sigma^{D} \cdot \varepsilon^{D}(\dot{u})\right]^{-}$is an $L^{2}\left(\Omega_{1}\right)$ function and 


$$
\int_{B}\left[\sigma^{D} \cdot \varepsilon^{D}(\dot{u})\right]^{-}=0
$$

for all subsets $B$ of $\Omega_{1}$ of Lebesgue measure zero; this holds in particular for all $B \subset \Gamma$ and we obtain from (2.8) that $(2.12)$ is true $\mathcal{H}^{2}$-almost everywhere in $\Gamma \cap C_{1}$. Taking into account (2.10), we see that (2.12) holds also $\mathcal{H}^{2}$-almost everywhere in $\Gamma$.

Now we prove (2.14). Recalls that, by definition,

$$
\varepsilon(\dot{u})=\frac{1}{3} \operatorname{div} \dot{u} I+\varepsilon^{D}(\dot{u})_{\perp}+\left[\sigma^{D} \cdot \varepsilon^{D}(\dot{u})\right]^{+} \frac{\sigma^{D}}{2 K^{2}}-\left[\sigma^{D} \cdot \varepsilon^{D}(\dot{u})\right]^{-} \frac{\sigma^{D}}{2 K^{2}} ;
$$

hence, for all Borel sets $B \subset \Omega_{1}$, it follows that

$$
\begin{aligned}
\frac{1}{\sqrt{2} K} \int_{B}\left[\sigma^{D} \cdot \varepsilon^{D}(\dot{u})\right]^{+} & \leqslant \int_{B}|\varepsilon(\dot{u})| \\
& \leqslant \int_{B}|\operatorname{div} \dot{u}|+\int_{B}\left|\varepsilon^{D}(\dot{u})_{\perp}\right|+\frac{1}{\sqrt{2} K} \int_{B}\left[\sigma^{D} \cdot \varepsilon^{D}(\dot{u})\right]^{+}+\frac{1}{\sqrt{2} K} \int_{B}\left[\sigma^{D} \cdot \varepsilon^{D}(\dot{u})\right]^{-}
\end{aligned}
$$

and this, when $B \subset \Gamma$, taking into account (2.8), yields (2.14).

Finally, from (2.5) and (2.14) we get

$$
\frac{1}{\sqrt{2}} \int_{B}\left|\dot{u}^{+}-\dot{u}^{-}\right| d \mathcal{H}^{2}=\frac{1}{\sqrt{2} K} \int_{B}\left[\left(\dot{u}^{+}-\dot{u}^{-}\right) \cdot L\right]^{+}
$$

for all Borel sets $B \subset \Gamma$, and (2.15) is also proved.

At this point we know from (2.15) that, $\mathcal{K}^{2}$-almost everywhere in $\Gamma$, we have $\left(\dot{u}^{+}-\dot{u}^{-}\right) \cdot L=0$ if and only if $\left(\dot{u}^{+}-\dot{u}^{-}\right)=0$, but we can tell more about $\dot{u}^{+}-\dot{u}^{-}$.

THEOREM 2.2. If $\dot{u} \in P_{\sigma}\left(\Omega_{1}\right)$, then we have

$$
\left(\dot{u}^{+}-\dot{u}^{-}\right)-\frac{1}{K^{2}}\left[\left(\dot{u}^{+}-\dot{u}^{-}\right) \cdot L_{t}\right] L_{t}=0
$$

$\mathcal{H}^{2}$-almost everywhere on $\Gamma$, and we have

$$
\begin{gathered}
\left|L_{t}(x)\right|=K, \\
\dot{u}^{+}-\dot{u}^{-}=\left|\dot{u}^{+}-\dot{u}^{-}\right| L_{t} / K
\end{gathered}
$$

for $\mathcal{H}^{2}$-almost all $x \in \Gamma$ such that $\left(\dot{u}^{+}-\dot{u}^{-}\right)(x) \neq 0$.

Proof. Set $\left(\dot{u}^{+}-\dot{u}^{-}\right)=v$. Take a point $x \in \Gamma$ such that

$$
\begin{aligned}
v(x) \cdot n(x) & =0, \\
\tau_{\perp}^{D}(v) & =0 .
\end{aligned}
$$

Choose a coordinate system in $\mathbb{R}^{3}$ such that $n(x)=(0,0,1)$, call $\left(v_{1}, v_{2}, v_{3}\right)$ the coordinates of $v(x)$ and set

$$
\sigma^{D}=\left(\begin{array}{lll}
a & d & e \\
d & b & f \\
e & f & c
\end{array}\right)
$$


Since $v \cdot n=0$ we have

$$
\tau^{D}(v)=\tau(v)=\left(\begin{array}{ccc}
0 & 0 & v_{1} / 2 \\
0 & 0 & v_{2} / 2 \\
v_{1} / 2 & v_{2} / 2 & 0
\end{array}\right)
$$

Condition (2.20) becomes

$$
\begin{aligned}
a\left(e v_{1}+f v_{2}\right) & =0, \\
b\left(e v_{1}+f v_{2}\right) & =0, \\
c\left(e v_{1}+f v_{2}\right) & =0, \\
d\left(e v_{1}+f v_{2}\right) & =0, \\
\frac{v_{1}}{2}-\frac{1}{2 K^{2}} e\left(e v_{1}+f v_{2}\right) & =0, \\
\frac{v_{2}}{2}-\frac{1}{2 K^{2}} f\left(e v_{1}+f v_{2}\right) & =0,
\end{aligned}
$$

and (2.16) follows at $x$, from the two last lines in (2.21).

Since $L_{t}=(e, f, 0)$ and $L \cdot v=L_{t} \cdot v=e v_{1}+f v_{2}$, if we assume that

$$
\begin{aligned}
L(x) \cdot \operatorname{ov}(x) & \neq 0, \\
& \left|\sigma^{D}(x)\right|=\sqrt{2} K,
\end{aligned}
$$

we obtain from (2.21) that $a=b=c=d=0$ and that

$$
\left|L_{t}\right|=\frac{1}{\sqrt{2}}\left|\sigma^{D}\right|=K,
$$

and (2.18) also follows.

To conclude the proof of the theorem it is sufficient to notice that conditions (2.20), (2.21) are satisfied $\mathcal{H}^{2}$-almost everywhere on $\Gamma$, and that, by (2.15) and (2.10), conditions (2.22), (2.23) are satisfied $\mathcal{H}^{2}$-almost everywhere in the set $\left\{x \in \Gamma \mid\left(\dot{u}^{+}-\dot{u}^{-}\right)(x) \neq 0\right\}$. Q.E.D.

We also have a glueing theorem, which is a sort of converse of Theorem 2.1.

THEOREM 2.3. Let $\dot{u} \in P_{\sigma}(\Omega)$ and let $\dot{g} \in P_{\sigma}\left(\Omega_{1} \backslash \bar{\Omega}\right)$ such that

$$
\begin{array}{rlrl}
\dot{u} & =\dot{g} & \mathcal{H}^{2} \text {-a.e. in }\left\{x \in \Gamma|| L_{t}(x) \mid<K\right\}, \\
\dot{u} \cdot n & =\dot{g} \cdot n \quad \mathscr{H}^{2} \text {-a.e. in } \Gamma, \\
{[(\dot{g}-\dot{u}) \cdot L]^{-}} & =0 \quad \mathcal{H}^{2} \text {-a.e. in } \Gamma, \\
\tau^{D}(\dot{g}-\dot{u}) & =[(\dot{g}-\dot{u}) \cdot L] \sigma^{D} \quad \mathscr{H}^{2} \text {-a.e. in } \Gamma .
\end{array}
$$

Then the function $\dot{v}$, defined by

$$
\dot{v}= \begin{cases}\dot{u} & \text { in } \Omega, \\ \dot{g} & \text { in } \Omega_{1} \backslash \bar{\Omega}\end{cases}
$$

belongs to $P_{\sigma}\left(\Omega_{1}\right)$. 
Proof. First, notice that $\dot{v} \in \mathrm{BD}\left(\Omega_{1}\right)$. In order to prove that $\operatorname{div}(\dot{v}) \in L^{2}\left(\Omega_{1}\right)$ it is sufficient to recall that for all $\varphi \in C_{0}^{\prime}\left(\Omega_{1}\right)$ we have

$$
\int_{\Omega_{1}} \varphi \operatorname{div}(\dot{v})=\int_{\Omega} \varphi \operatorname{div}(\dot{u})+\int_{\Gamma}(\dot{g}-\dot{u}) \cdot n \varphi+\int_{\Omega_{1} \backslash \bar{\Omega}} \varphi \operatorname{div}(\dot{g})
$$

where $(\dot{g}-\dot{u}) \cdot n=0$ on $\Gamma$ by $(2.24 i i)$; hence it follows that $\operatorname{div}(\dot{v})=\eta \in L^{2}\left(\Omega_{1}\right)$, where we have set

$$
\eta= \begin{cases}\operatorname{div}(\dot{u}) & \text { in } \Omega, \\ \operatorname{div}(\dot{g}) & \text { in } \Omega_{1} \backslash \bar{\Omega} .\end{cases}
$$

Now we want to prove that $\varepsilon^{D}(\dot{v})_{\perp}$ is in $L^{2}\left(\Omega_{1}\right)$. We have, by additivity of measures and by $(2.9)$,

$$
\begin{aligned}
\int_{\Omega_{1}} \varphi \varepsilon_{i j}^{D}(\dot{v})_{\perp}= & \int_{\Omega} \varphi \varepsilon_{i j}^{D}(\dot{u})_{\perp}+\int_{\Omega_{1} \backslash \bar{\Omega}} \varphi \varepsilon_{i j}^{D}(\dot{g})_{\perp} \\
& +\int_{\Gamma \backslash C_{1}} \varphi \tau_{i j}^{D}(\dot{g}-\dot{u}) d \mathcal{H}^{2}+\int_{\Gamma \cap C_{1}} \varphi\left\{\tau_{i j}^{D}(\dot{g}-\dot{u})-[(\dot{g}-\dot{u}) \cdot L] \sigma_{i j}^{D}\right\} d \mathcal{H}^{2}
\end{aligned}
$$

where the last two terms are zero because of $\left(2.24 \mathrm{i}\right.$, and iii); hence we have $\varepsilon_{i j}^{D}(\dot{v}) \in L^{2}\left(\Omega_{1}\right)$ for all $i, j=1,2,3$.

Finally, we have from (2.8)

$$
\int_{\Omega_{1}} \varphi\left(\sigma^{D} \cdot \varepsilon^{D}(\dot{v})\right)^{-}=\int_{\Omega} \varphi\left(\sigma^{D} \cdot \varepsilon^{D}(\dot{u})\right)^{-}+\int_{\Gamma} \varphi[(\dot{g}-\dot{u}) \cdot L]^{-} d \mathcal{H}^{2}+\int_{\Omega_{1} \backslash \bar{\Omega}} \varphi\left(\sigma^{D} \cdot \varepsilon^{D}(\dot{g})\right)^{-}
$$

and by $\left(2.24\right.$ iv) we get also $\left(\sigma^{D} \cdot \varepsilon^{D}(\dot{v})\right)^{-} \in L^{2}\left(\Omega_{1}\right)$. Q.E.D.

3. In this section we are going to give a meaning to the expressions

$$
\int_{\Omega} \sigma_{i j} \varepsilon_{i j}(u), \quad \int_{\partial \Omega} \sigma_{i j} n_{j} u_{i}
$$

for $\sigma \in \Sigma(\Omega), u \in P(\Omega)$, where

$$
\begin{aligned}
P(\Omega) & =\left\{u \in \operatorname{BD}(\Omega) \mid \operatorname{div} u \in L^{2}(\Omega)\right\}, \\
\Sigma(\Omega) & =\left\{\sigma: \Omega \rightarrow \mathbb{R}^{9} \mid \sigma=\left\{\sigma_{i j}\right\}_{i, j=1,2,3}, \sigma_{i j}\right. \text { are measurable, } \\
\sigma_{i j} & \left.=\sigma_{j i}, \text { trace } \sigma \in L^{2}(\Omega),\left\|\sigma^{D}\right\|_{\infty, \Omega} \leqslant \sqrt{2} K, \sigma_{i j, j} \in L^{3}(\Omega)\right\} .
\end{aligned}
$$

The derivatives $\sigma_{i j, j}$ are intended in the sense of distributions and we set $\operatorname{div} \sigma=$ $\left\{\sigma_{i j, j}\right\}_{i=1,2,3}$. For the results in this section compare also with [6].

We shall consider the space of displacement fields

$$
L P(\Omega)=\left\{u \in P(\Omega) \mid \varepsilon_{i j}(u) \in L^{1}(\Omega)\right\}
$$

and we remark that $P(\Omega) \cap H_{\text {loc }}^{1,1}(\Omega) \subset L P(\Omega)$.

We shall need the following lemma. 
LEMma 3.1. Assume that $\Omega$ has a Lipschitz boundary. If $w \in L P(\Omega)$ and the trace of $w$ on $\partial \Omega$ is zero, then there exists a sequence of functions $g_{h} \in C_{0}^{\infty}\left(\Omega, \mathbb{R}^{3}\right)$ such that

$$
\begin{aligned}
g_{h} & \rightarrow w \quad \text { in } L^{3 / 2}(\Omega), \\
\varepsilon\left(g_{h}\right) & \rightarrow \varepsilon(w) \quad \text { in } L^{1}(\Omega), \\
\operatorname{div} g_{h} & \rightarrow \operatorname{div} w \quad \text { in } L^{2}(\Omega) .
\end{aligned}
$$

Proof. By standard techniques in Sobolev space theory, we know that there exists a sequence of functions $w_{h} \in C_{0}^{\infty}(\Omega)$ such that

$$
\begin{aligned}
w_{h} & \rightarrow w \quad \text { in } L^{3 / 2}(\Omega), \\
\varepsilon\left(w_{h}\right) & \rightarrow \varepsilon(w) \quad \text { in } L^{1}(\Omega), \\
\operatorname{div} w_{h} & \rightarrow \operatorname{div} w \quad \text { in } L^{3 / 2}(\Omega) .
\end{aligned}
$$

Take then a sequence $\eta_{h} \in C_{0}^{\infty}(\Omega)$ such that

$$
\begin{aligned}
& \eta_{h} \rightarrow \operatorname{div} w \quad \text { in } L^{2}(\Omega), \\
& \int_{\Omega}\left(\eta_{h}-\operatorname{div} w_{h}\right)=0 \quad \text { for all } h
\end{aligned}
$$

and, for every $h \in \mathbb{N}$, let $f_{h} \in C_{0}^{\infty}(\Omega)$ be such that ([3], remark 4)

$$
\operatorname{div} f_{h}=\eta_{h}-\operatorname{div} w_{h}, \quad\left\|f_{h}\right\|_{H^{1,3 / 2}} \leqslant C\left\|\eta_{h}-\operatorname{div} w_{h}\right\|_{L^{3 / 2}} .
$$

Now the lemma is proved just by choosing $g_{h}=w_{h}+f_{h}$.

Now we shall give a meaning to $\sigma_{i j} n_{j}$ on $\partial \Omega$.

THEOREM 3.1. There exists a bilinear form

$$
\langle\sigma, u\rangle_{\partial \Omega}: \Sigma(\Omega) \times P(\Omega) \rightarrow \mathbb{R}
$$

such that

$$
\begin{gathered}
\langle\sigma, u\rangle_{\partial \Omega}=\int_{\partial \Omega} \sigma_{i j} n_{j} u_{i} \text { if } \sigma \text { and } u \text { are smooth in } \bar{\Omega}, \\
\left|\langle\sigma, u\rangle_{\partial \Omega}\right| \leqslant\left\|\sigma^{D}\right\|_{\infty} \int_{\Omega}\left|\varepsilon^{D}(u)\right|+\frac{1}{3}\|\operatorname{trace} \sigma\|_{L^{2}}\|\operatorname{div} u\|_{L^{2}}+\|\operatorname{div} \sigma\|_{L^{3}}\|u\|_{L^{3 / 2}} \\
\text { for all }(\sigma, u) \in \Sigma(\Omega) \times P(\Omega) .
\end{gathered}
$$

Moreover, if $\Omega$ has a class $C^{2}$ boundary, one can prove that

$$
\left|\langle\sigma, u\rangle_{\partial \Omega}\right| \leqslant \frac{1}{\sqrt{2}}\left\|\sigma^{D}\right\|_{\infty} \int_{\partial \Omega}|u| d \mathcal{H}^{2}
$$

for all $\sigma \in \Sigma(\Omega)$ and for all $u \in P(\Omega)$ such that $u \cdot n=0$ on $\partial \Omega$.

Proof. In order for (3.1) to be satisfied, we shall define

$$
\langle\sigma, u\rangle_{\partial \Omega}=\int_{\Omega} \sigma_{i j, j} u_{i}+\int_{\Omega} \sigma_{i j}^{D} \varepsilon_{i j}^{D}(u)+\frac{1}{3} \int_{\Omega} \operatorname{trace} \sigma \operatorname{div} u
$$

for all $\sigma \in \Sigma(\Omega)$ and for all $u \in L P(\Omega)$ (notice that the right member of (3.4) wouldn't 
have a defined meaning if $\varepsilon^{D}(u)$ were only a measure) and we have that (3.2) holds in this case; moreover $\langle\sigma, u\rangle_{\partial \Omega}$ is clearly bilinear.

Now, observe that, if $u, v \in L P(\Omega)$, one has

$$
\langle\sigma, u\rangle_{\partial \Omega}=\langle\sigma, v\rangle_{\partial \Omega} \quad \text { if } u=v \text { on } \partial \Omega .
$$

In fact, take a sequence $g_{h} \in C_{0}^{\infty}(\Omega)$ approximating $w=u-v$ as in Lemma 3.1. By (3.2), we have $\left\langle\sigma, w-g_{h}\right\rangle_{\partial \Omega} \rightarrow 0$ and since $\sigma_{i j, j}$ are derivatives in the sense of distributions we have also $\left\langle\sigma, g_{h}\right\rangle_{\partial \Omega}=0$ for all $h \in \mathbb{N}$ and (3.5) is proved.

Now, we are able to define $\langle\sigma, u\rangle_{\partial \Omega}$ for all $u \in P(\Omega)$ by setting

$$
\langle\sigma, u\rangle_{\partial \Omega}=\langle\sigma, v\rangle_{\partial \Omega}
$$

where $v$ is any function in $L P(\Omega)$ with $v=u$ on $\partial \Omega$. This is a valid definition because (i) $\langle\sigma, v\rangle_{\partial \Omega}$ depends only on $\left.v\right|_{\partial \Omega}$ by (3.5); (ii) for all $u \in P(\Omega)$ there exists a function $v \in L P(\Omega)$ with $v=u$ on $\partial \Omega$ (see Theorem 5.1 in the appendix of [2]).

In order to prove (3.2), take fixed $\sigma, u$ and let $v_{h} \in P(\Omega) \cap H_{\text {loc }}^{1,1}(\Omega)$ be a sequence of functions approximating $u$ as in Theorem 5.1 of [2], i.e. such that

$$
\begin{gathered}
v_{h} \rightarrow u \text { in } L^{3 / 2}(\Omega), \\
\int_{\Omega}\left|\varepsilon\left(v_{h}\right)\right| \rightarrow \int_{\Omega}|\varepsilon(u)|, \\
\operatorname{div} v_{h} \rightarrow \operatorname{div} u \quad \text { in } L^{2}(\Omega), \\
\left.v_{h}\right|_{\partial \Omega}=\left.u\right|_{\partial \Omega} .
\end{gathered}
$$

For all $h$ we have $\langle\sigma, u\rangle_{\partial \Omega}=\left\langle\sigma, v_{h}\right\rangle_{\partial \Omega}$ and, since (3.2) holds for all $v_{h}$, it also holds at the limit because of (3.6).

Finally, estimate (3.3) follows from (3.2) because, for all $u \in L^{1}(\partial \Omega)$ with $u \cdot n=0$ and for any given $\rho>0$, there exists (Theorem 5.2 in [2]) a function $v \in P(\Omega) \cap H_{\mathrm{loc}}^{1,1}(\Omega)$ such that

$$
\begin{array}{r}
\int_{\Omega}\left|\varepsilon^{D}(v)\right| \leqslant \frac{1}{\sqrt{2}} \int_{\partial \Omega}|u|+\rho, \\
\|\operatorname{div} v\|_{L^{2}} \leqslant \rho, \quad\|u\|_{L^{3 / 2}} \leqslant \rho,
\end{array}
$$

so that we have for all $\rho>0$

$$
\left|\langle\sigma, u\rangle_{\partial \Omega}\right| \leqslant|\langle\sigma, v\rangle|_{\partial \Omega} \leqslant \frac{1}{\sqrt{2}}\left\|\sigma^{D}\right\|_{\infty} \int_{\Omega}|u|+3 \rho C(\sigma) \text {. Q.E.D. }
$$

Remark 3.1. Theorem 3.1 says that, for all $\sigma \in \Sigma(\Omega)$, the trace $\sigma_{i j} n_{j}$ on $\partial \Omega$ belongs to the dual space of the traces of functions in $P(\Omega)$ (or in $P(\Omega) \cap H_{\text {loc }}^{1,1}(\Omega)$, or in $L P(\Omega)$ ). As far as I know, it is an open question whether or not every "vector field" in this dual corresponds to $\alpha_{i j} n_{j}$ for some $\alpha \in \Sigma(\Omega)$.

Now we shall give a meaning to $\int_{\Omega} \sigma_{i j} \varepsilon_{i j}(u)$.

THEOREM 3.2. There exists a bilinear form

$$
\langle\sigma, u\rangle_{\Omega}: \Sigma(\Omega) \times P(\Omega) \rightarrow \mathbb{R}
$$


such that

$$
\begin{gathered}
\langle\sigma, u\rangle_{\Omega}=\int_{\Omega} \sigma_{i j} \varepsilon_{i j}(u) \quad \text { if } u \in L P(\Omega), \\
\left|\langle\sigma, u\rangle_{\Omega}\right| \leqslant \sqrt{2} K \int_{\Omega}\left|\varepsilon^{D}(u)\right|+\frac{1}{3}\|\operatorname{trace} \sigma\|_{L^{2}}\|\operatorname{div} u\|_{L^{2}}
\end{gathered}
$$

for all $(\sigma, u) \in \Sigma(\Omega) \times P(\Omega)$.

Proof. For all $\sigma \in \Sigma(\Omega)$ and for all $u \in P(\Omega)$ define

$$
\langle\sigma, u\rangle_{\Omega}=-\int_{\Omega} \sigma_{i j, j} u_{i}+\langle\sigma, u\rangle_{\partial \Omega}
$$

Obviously, $\langle\sigma, u\rangle_{\Omega}$ is bilinear and (3.7) holds by the definition of $\langle\sigma, u\rangle_{\Omega}$. In order to get (3.8), take a sequence of functions $v_{h} \in P(\Omega) \cap H_{l o c}^{1,1}(\Omega)$ converging to $u$ as in (3.6) and notice that

$$
\begin{aligned}
\left\langle\sigma, v_{h}\right\rangle_{\Omega} & =-\int_{\Omega} \operatorname{div} \sigma \cdot v_{h}+\left\langle\sigma, v_{h}\right\rangle_{\partial \Omega} \\
& =-\int_{\Omega} \operatorname{div} \sigma \cdot v_{h}+\langle\sigma, u\rangle_{\partial \Omega} \rightarrow\langle\sigma, u\rangle_{\Omega}
\end{aligned}
$$

while for all $h \in \mathbb{N}$ we have

$$
\left|\left\langle\sigma, v_{h}\right\rangle_{\Omega}\right| \leqslant \sqrt{2} K \int_{\Omega}\left|\varepsilon^{D}\left(v_{h}\right)\right|+\frac{1}{3}\|\operatorname{trace} \sigma\|_{L^{2}}\left\|\operatorname{div} v_{h}\right\|_{L^{2}}
$$

and (3.8) follows taking the limit for $h \rightarrow \infty$.

Remark 3.2. In the proof of Theorem 3.2 we have used the following fact that we want to point out explicitly: if $v_{h} \in P(\Omega) \cap H_{\text {loc }}^{1,1}(\Omega)$ converge to $u \in P(\Omega)$ as in (3.6), one has that $\left\langle\sigma, v_{h}\right\rangle_{\Omega} \rightarrow\langle\sigma, u\rangle_{\Omega}$. Since any function $u \in P(\Omega)$ can be approximated by a sequence $v_{h}$ as in (3.6), one could use the preceding property to define $\langle\sigma, u\rangle_{\Omega}$.

Now we shall see how formulae (3.1), (3.7) work in the case that some continuity is assumed for $\sigma^{D}$.

TheOREM 3.3. Let $\sigma \in \Sigma(\Omega)$ such that $\sigma^{D} \in C^{0}(\Omega)$; then we have

$$
\langle\sigma, u\rangle_{\Omega}=\int_{\Omega} \sigma^{D} \cdot \varepsilon^{D}(u)+\frac{1}{3} \int_{\Omega} \operatorname{trace} \sigma \operatorname{div} u
$$

for all $u \in P(\Omega)$.

Proof. Take a sequence $v_{h} \in P(\Omega) \cap H_{\text {loc }}^{1,1}(\Omega)$ approximating $u$ as in (3.6); by Remark 3.2 and by (3.7) we have

$$
\langle\sigma, u\rangle_{\Omega}=\lim _{h \rightarrow \infty}\left\{\int_{\Omega} \sigma^{D} \cdot \varepsilon^{D}\left(v_{h}\right)+\frac{1}{3} \int_{\Omega} \operatorname{trace} \sigma \operatorname{div} v_{h}\right\} .
$$

Observe that the convergence (3.6) implies in particular the weak convergence of the measures $\varepsilon^{D}\left(v_{h}\right)$ to $\varepsilon^{D}(u)$, i.e.

$$
\int_{\Omega} \psi_{i j} \varepsilon_{i j}^{D}\left(v_{h}\right) \rightarrow \int_{\Omega} \psi_{i j} \varepsilon_{i j}^{D}(u) \text { for all } \psi \in C_{0}^{0}(\Omega)^{9}
$$


Take a fixed number $\delta>0$ and let $V$ be an open set such that

$$
V \subset \subset \Omega, \quad \int_{\partial V}\left|\varepsilon^{D}(u)\right|=0, \quad \int_{\Omega \backslash V}\left|\varepsilon^{D}(u)\right|<0
$$

and consequently such that

$$
\lim _{h \rightarrow \infty} \int_{\Omega \backslash V}\left|\varepsilon^{D}\left(v_{h}\right)\right| \leqslant \delta .
$$

Now, if $\varphi \in C_{0}^{0}(\Omega), 0 \leqslant \varphi \leqslant 1, \varphi=1$ in $V$, we have

$$
\begin{aligned}
\lim _{h \rightarrow \infty} \int_{\Omega}\left\{\sigma^{D} \cdot \varepsilon\left(v_{h}\right)-\sigma^{D} \cdot \varepsilon^{D}(u)\right\} \\
\quad=\lim _{h \rightarrow \infty} \int_{\Omega}(1-\varphi) \sigma^{D} \cdot\left(\varepsilon^{D}\left(v_{h}\right)-\varepsilon^{D}(u)\right)+\lim _{h \rightarrow \infty} \int_{\Omega} \varphi \sigma^{D} \cdot\left(\varepsilon^{D}\left(v_{h}\right)-\varepsilon^{D}(u)\right)
\end{aligned}
$$

where, by (3.11) and (3.11)', the first limit on the right side is less than $2 \sqrt{2} K \delta$ and the second limit is zero by (3.10). Because $\delta>0$ is arbitrary, we have proved that

$$
\lim _{h \rightarrow \infty} \int_{\Omega} \sigma \cdot \varepsilon^{D}\left(v_{h}\right)=\int_{\Omega} \sigma^{D} \cdot \varepsilon^{D}(u)
$$

and, as from (3.6) it follows obviously that

$$
\lim _{h \rightarrow \infty} \int_{\Omega} \operatorname{trace} \sigma \operatorname{div} v_{h}=\int_{\Omega} \operatorname{trace} \sigma \operatorname{div} v,
$$

the theorem is proved by (3.9). Q.E.D.

We need the following lemma to prove Theorem 3.4.

LeMmA 3.2. Let $\Omega_{1}$ be an open set such that $\Omega_{1} \supset \supset \Omega$ and let $\sigma \in \Sigma\left(\Omega_{1}\right)$ such that $\sigma^{D} \in C^{0}(\bar{\Omega})$; then there exists a sequence $\alpha^{h} \in \Sigma(\Omega) \cap C^{1}(\bar{\Omega})$ such that

$$
\begin{aligned}
\left(\alpha^{h}\right)^{D} & \rightarrow \sigma^{D} \quad \text { uniformly on } \bar{\Omega}, \\
\operatorname{trace} \alpha^{h} & \rightarrow \operatorname{trace} \sigma \quad \text { in } L^{2}(\Omega), \\
\alpha_{i j, j}^{h} & \rightarrow \sigma_{i j, j} \quad \text { in } L^{3}(\Omega)
\end{aligned}
$$

Proof. Just take a sequence of mollifiers $\psi_{h}$ in $\mathbb{R}^{3}$ with $\operatorname{diam}\left(\operatorname{spt}\left(\psi_{h}\right)\right)<\operatorname{dist}\left(\bar{\Omega}, \partial \Omega_{1}\right)$ and set, for all $h$,

$$
\alpha_{i j}^{h}=\sigma_{i j} * \psi_{h} \text { for } i, j=1,2,3 \text {. Q.E.D. }
$$

THEOREM 3.4. Let $\Omega_{1}$ be an open set in $\mathbb{R}^{3}$ such that $\Omega \subset \subset \Omega_{1}$, let $\sigma \in \Sigma\left(\Omega_{1}\right)$ such that $\sigma^{D} \in C^{0}\left(\Omega_{1}\right)$ and set $L=\left(L_{1}, L_{2}, L_{3}\right), L_{t}=L-(L \cdot n) n$, where

$$
L_{i}(x)=\sigma_{i j}^{D}(x) n_{j}(x) \text {. }
$$

Then we have

$$
\langle\sigma, u\rangle_{\partial \Omega}=\int_{\partial \Omega} L_{t} \cdot u
$$

for all $u \in P(\Omega)$ such that $u \cdot n=0$ on $\partial \Omega$. 
Proof. Take a sequence $\alpha^{h} \in \Sigma(\Omega) \cap C^{1}(\bar{\Omega})$ approximating $\sigma$ as in Lemma 3.2. For all $h$ we have, by Green's formula (Theorem 1.4),

$$
\int_{\partial \Omega} \alpha_{i j}^{h} n_{j} u_{i}=\int_{\Omega}\left(\alpha^{h}\right)_{i j}^{D} \varepsilon_{i j}(u)+\frac{1}{3} \int_{\Omega} \operatorname{trace} \alpha^{h} \operatorname{div} u+\int_{\Omega} \alpha_{i j, j}^{h} u_{i}
$$

where, as $u \cdot n=0$ on $\partial \Omega$,

$$
\int_{\partial \Omega} \alpha_{i j}^{h} n_{j} u_{i}=\int_{\partial \Omega}\left(\alpha^{h}\right)_{i j}^{D} n_{j} u_{i} .
$$

Taking the limit in (3.14) for $h \rightarrow \infty$, and also using Theorem 3.3, we obtain

$$
\int_{\partial \Omega} \sigma_{i j}^{D} n_{j} u_{i}=\langle\sigma, u\rangle_{\partial \Omega}
$$

where, again because $u \cdot n=0$ on $\partial \Omega$,

$$
\int_{\partial \Omega} \sigma_{i j}^{D} n_{j} u_{i}=\int_{\partial \Omega} L_{t} \cdot u \text {. Q.E.D. }
$$

4. In this and the following section, we shall study problem (P.1) in the case $\Gamma_{D}=\partial \Omega$, $\Gamma_{N}=\varnothing$, that is, we study the Dirichlet problem for $\dot{u}$. More precisely, in this section we shall give an existence theorem for problem (P.2) and in the next section we shall see how this yields a (necessarily weak) solution to problem (P.1).

We shall need the following assumptions on the given state of stress $\sigma$ : there exists an open set $\Omega_{1} \supset \supset \Omega$ such that $\sigma$ is defined also in $\Omega_{1}, \sigma$ satisfies hypotheses (H.1.1) in $\Omega_{1}$, and the distributions $\sigma_{i j, j}$ are $L^{3}$ functions in $\Omega_{1}$ (hence we have also $\sigma \in \Sigma\left(\Omega_{1}\right)$ ). Moreover, we shall assume that

(H.4.1) there exists a stress field $\alpha \in \Sigma(\Omega)$ such that

$$
\langle\alpha, \varphi\rangle_{\Omega}=\langle\sigma, \varphi\rangle_{\Omega} \text { for all } \varphi \in P(\Omega)
$$

and that $\left\|\alpha^{D}\right\|_{\infty}<\sqrt{2} K$.

Notice that (4.1) implies in particular that $\alpha_{i j, j}=\sigma_{i j, j}$ in $\Omega$ in the sense of distributions and that

$$
\langle\sigma, u\rangle_{\partial \Omega}=\langle\alpha, u\rangle_{\partial \Omega} \text { for all } u \in P(\Omega) .
$$

Formula (4.1)' is just a weak formulation of the condition

$$
\sigma_{i j} n_{j}=\alpha_{i j} n_{j} \text { on } \partial \Omega \text {. }
$$

In conclusion, formula (4.1) says that $\sigma$ and $\alpha$ are in equilibrium with the same system of forces.

If (H.4.1) holds, we say that the load state of the body associated with $\sigma$ is safe.

Remark 4.1. Obviously, the load state is safe if $\left\|\sigma^{D}\right\|_{\infty}<\sqrt{2} K$, but, in such a case, the body would behave in a purely elastic way. It seems to be natural, however, to have an elasto-plastic stress state $\sigma\left(\sigma^{D}(x)=\sqrt{2} K\right.$ in some part of $\left.\Omega\right)$ which is in equilibrium with a safe load (compare also with Theorem 1.1 in [2]).

We shall consider the following problem (later we shall take into account also the body forces $\dot{f}$ ): 
Problem (P.2). Given a stress field $\sigma \in \Sigma\left(\Omega_{1}\right)$ satisfying (H.1.1) in $\Omega_{1}$ and (H.4.1), and given a function $\dot{g} \in P_{\sigma}\left(\Omega_{1}\right) \cap H_{\mathrm{loc}}^{1,1}\left(\Omega_{1}\right)$, find a minimum point for the functional

$$
E_{1}(\dot{u})=\frac{K_{0}}{2} \int_{\Omega}(\operatorname{div} \dot{u})^{2} d x+\mu \int_{\Omega}\left|e^{D}(\dot{u})\right|^{2} d x
$$

among the functions $\dot{u} \in P_{\sigma}(\Omega)$ such that

$$
\left.\begin{array}{rl}
\dot{u} & =\dot{g} \quad \mathcal{K}^{2} \text {-a.e. in }\left\{x \in \partial \Omega|| L_{t}(x) \mid<K\right\}, \\
\dot{u} \cdot n & =\dot{g} \cdot n \\
\tau^{D}(\dot{g}-\dot{u})-[(\dot{g}-\dot{u}) \cdot L] \sigma^{D} & =0 \\
{[(\dot{g}-\dot{u}) \cdot L]^{-}} & =0
\end{array}\right\} \mathcal{K}^{2} \text {-a.e. on } \partial \Omega .
$$

Notice that the two traces $\dot{g}^{+}$and $\dot{g}^{-}$of $\dot{g}$ on $\partial \Omega$ coincide because $g \in H_{\mathrm{loc}}^{1,1}\left(\Omega_{1}\right)$, so that we can talk of the trace of $\dot{g}$ on $\partial \Omega$.

Remark 4.2. A remark is in order about the fact that both $\sigma$ and $\dot{g}$ are required to be given in a larger set than $\Omega$. Of course, one would like to be able to work without this assumption and this could be accomplished, for example, if one had suitable extension theorems for functions of $\Sigma(\Omega)$ and of $P_{\sigma}(\Omega)$ (at least in the case of a smooth $\partial \Omega$ ). It should be noticed, however, that, if $\sigma$ and $\dot{g}$ are Lipschitz-continuous functions in $\Omega$, they can be extended as required. Notice also that for the Neumann problem (see Sec. 6), we shall require only that $\sigma$ be defined in $\Omega$.

To show the existence of a solution to Problem (P.2), we shall use the direct method of calculus of variations; and we shall need suitable compactness, coerciveness, semicontinuity results which we shall prove first.

Set $U=\left\{\dot{u} \in P_{\sigma}(\Omega) \mid \dot{u}\right.$ satisfies conditions (4.2) $\}$.

TheOREM 4.1. Given a stress field $\sigma \in \Sigma\left(\Omega_{1}\right)$ satisfying (H.1.1) in $\Omega_{1}$ and (H.4.1), and given a function $\dot{g} \in P_{\sigma}\left(\Omega_{1}\right)$, there exist two positive numbers $c$ and $M$, depending on $\sigma, g$, $\Omega, K, K_{0}, \mu$ (but not on $\dot{u}$ ) such that

$$
E_{1}(\dot{u}) \geqslant c\|u\|_{P_{o}(\Omega)}-M
$$

for all $\dot{u} \in U$. We say then that $E_{1}(\dot{u})$ is coercive on $U$.

Proof. Take $\dot{u} \in U$; then, by Theorem 3.4, we have

$$
\langle\sigma, \dot{g}-\dot{u}\rangle_{\partial \Omega}=\int_{\partial \Omega}(\dot{g}-\dot{u}) \cdot L_{t} ;
$$

by Theorem 2.1 we have

$$
\int_{\partial \Omega}(\dot{g}-\dot{u}) \cdot L_{t}=K \int_{\partial \Omega}|\dot{g}-\dot{u}| ;
$$

and by Theorem 3.3, recalling Definition 1.4, we get

$$
\langle\sigma, \dot{u}\rangle_{\Omega}=\int_{\Omega} \sigma^{D} \cdot e^{D}(\dot{u})+\int_{\Omega}\left[\sigma^{D} \cdot \varepsilon^{D}(\dot{u})\right]^{+}+\frac{1}{3} \int_{\Omega} \operatorname{trace} \sigma \operatorname{div} \dot{u} .
$$


It follows that

$$
\begin{aligned}
E_{1}(\dot{u})= & \frac{K_{0}}{2} \int_{\Omega}(\operatorname{div} \dot{u})^{2}+\mu \int_{\Omega}\left|e^{D}(\dot{u})\right|^{2}-\langle\sigma, \dot{u}\rangle_{\Omega}-\langle\sigma, \dot{g}-\dot{u}\rangle_{\partial \Omega} \\
& +\int_{\Omega} \sigma^{D} \cdot e^{D}(\dot{u})+\int_{\Omega}\left[\sigma^{D} \cdot \varepsilon^{D}(\dot{u})\right]^{+} \\
& +\frac{1}{3} \int_{\Omega} \operatorname{trace} \sigma \operatorname{div} \dot{u}+K \int_{\partial \Omega}|\dot{g}-\dot{g}|
\end{aligned}
$$

where, by the safe load assumption (H.4.1) and by Theorems 3.1, 3.2, we have

$$
\begin{aligned}
& \left|\langle\sigma, \dot{u}\rangle_{\Omega}\right|=\left|\langle\alpha, \dot{u}\rangle_{\Omega}\right| \leqslant\left(1-C_{0}\right) \sqrt{2} K \int_{\Omega}\left|\varepsilon^{D}(\dot{u})\right|+\frac{1}{3}\|\operatorname{trace} \alpha\|_{L^{2}}\|\operatorname{div} \dot{u}\|_{L^{2}}, \\
& \left|\langle\sigma, \dot{u}\rangle_{\partial \Omega}\right|=\left|\langle\alpha, \dot{u}\rangle_{\partial \Omega}\right| \leqslant\left(1-C_{0}\right) K \int_{\partial \Omega}|\dot{g}-\dot{u}| d \mathcal{H}^{2},
\end{aligned}
$$

where $0<C_{0}<1$. Now, by the Hölder inequality and recalling that

$$
\int_{\Omega}\left|\varepsilon^{D}(\dot{u})\right| \leqslant \int_{\Omega}\left|e^{D}(\dot{u})\right|+\frac{1}{\sqrt{2} K} \int_{\Omega}\left[\sigma^{D} \cdot \varepsilon^{D}(\dot{u})\right]^{+}
$$

we obtain

$$
\begin{aligned}
E_{1}(\dot{u}) \geqslant & \frac{K_{0}}{4} \int_{\Omega}(\operatorname{div} \dot{u})^{2}+\frac{\mu}{4} \int_{\Omega}\left|e^{D}(\dot{u})\right|^{2}+\sqrt{2} K C_{0} \int_{\Omega}\left|\varepsilon^{D}(\dot{u})\right| \\
& +C_{0} K \int_{\partial \Omega}|\dot{u}-\dot{g}| d \mathcal{H}^{2}-M_{1}\left(K_{0}, \mu, K, \alpha, g, \Omega\right)
\end{aligned}
$$

and, using the Poincare inequality (Theorem 1.2) to estimate $\|u\|_{L^{1}}$, the theorem follows. Q.E.D.

Remark 4.3. If $E_{1}(\dot{u})$ is coercive on $U$ and $\|\dot{f}\|_{L^{3}}$ is sufficiently small, then the functional

$$
E_{1}(\dot{u})-\int_{\Omega} \dot{f} \dot{u}
$$

is also coercive on $U$. In fact, one has

$$
\left|\int_{\Omega} \dot{f} \dot{u}\right| \leqslant\|\dot{f}\|_{L^{3}}\|\dot{u}\|_{L^{3 / 2}} \leqslant C\|\dot{f}\|_{L^{3}}\|\dot{u}\|_{P_{\sigma}(\Omega)}
$$

for all $\dot{u} \in P_{\sigma}(\Omega)$.

Definition. 4.1. We shall say that a sequence of functions $\dot{u}_{h} \in P_{\sigma}(\Omega)$ converges $P_{\sigma}(\Omega)$-weakly to $\dot{u} \in P_{\sigma}(\Omega)$ if

$$
\dot{u}_{h} \rightarrow \dot{u} \quad \text { in } L^{1}(\Omega) \text { and weakly in } L^{3 / 2}(\Omega),
$$

$\operatorname{div} \dot{u}_{h} \rightarrow \operatorname{div} \dot{u} \quad$ weakly in $L^{2}(\Omega)$,

$\varepsilon^{D}\left(\dot{u}_{h}\right)_{\perp} \rightarrow \varepsilon^{D}(\dot{u})_{\perp} \quad$ weakly in $L^{2}(\Omega)$,

$\left[\sigma^{D} \cdot \varepsilon^{D}\left(\dot{u}_{h}\right)\right] \rightarrow\left[\sigma^{D} \cdot \varepsilon^{D}(\dot{u})\right]$ weakly as measures in $\Omega$, 
and if there exist a non-negative function $\gamma(x) \in L^{2}(\Omega)$ and a positive measure $\mu_{1}$ in $\Omega$ such that

$$
\begin{aligned}
& {\left[\sigma^{D} \cdot \varepsilon^{D}\left(\dot{u}_{h}\right)\right]^{-} \rightarrow \gamma \quad \text { weakly in } L^{2}(\Omega),} \\
& {\left[\sigma^{D} \cdot \varepsilon^{D}\left(\dot{u}_{h}\right)\right]^{+} \rightarrow \mu_{1} \quad \text { weakly as measures in } \Omega .}
\end{aligned}
$$

Remark 4.4. If $\dot{u}_{h} \rightarrow \dot{u} P_{\sigma}(\Omega)$-weakly, then

$$
\gamma(x) \geqslant\left[\sigma^{D} \cdot \varepsilon^{D}(\dot{u})\right]^{-}(x) \text { a.e. in } \Omega .
$$

In fact, if we consider the positive measure $\mu_{2}(B)=\int_{B} \gamma(x) d x$, we have

$$
\left[\sigma^{D} \cdot \varepsilon^{D}(\dot{u})\right]^{+}-\left[\sigma^{D} \cdot \varepsilon^{D}(\dot{u})\right]^{-}=\mu_{1}-\mu_{2} ;
$$

hence $\left[\sigma^{D} \cdot \varepsilon^{D}(\dot{u})\right]^{-} \leqslant \mu_{2}$ and the same holds for the densities.

Now we shall give a compactness theorem.

THEOREM 4.2. Let $\dot{u}_{h} \in P_{\sigma}(\Omega)$ be such that

$$
\left\|\dot{u}_{h}\right\|_{P_{o}(\Omega)} \leqslant c<+\infty \text { for all } h \in \mathbb{N} ;
$$

then there exists a subsequence $u_{h_{j}}$ that converges $P_{\sigma}(\Omega)$-weakly to some $u \in P_{\sigma}(\Omega)$.

Proof. If the sequence $\dot{u}_{h}$ is bounded in $P_{\sigma}(\Omega)$, it is bounded also in $\operatorname{BD}(\Omega)$ and, by Theorem 1.3, possibly taking a subsequence, we have

$$
\dot{u}_{h} \rightarrow \dot{u} \text { in } L^{1}(\Omega)
$$

for some function $u \in \mathrm{BD}(\Omega)$. From this it follows in particular that

$$
\begin{aligned}
\int_{\Omega} \dot{u}_{h} \cdot \varphi & \rightarrow \int_{\Omega} \dot{u} \cdot \varphi \text { for all } \varphi \in C_{0}^{\infty}\left(\Omega, \mathbb{R}^{3}\right), \\
\int_{\Omega} \psi \operatorname{div} \dot{u}_{h} & \rightarrow \int_{\Omega} \psi \operatorname{div} \dot{u} \text { for all } \psi \in C_{0}^{\infty}(\Omega),
\end{aligned}
$$

and, as the norms $\left\|\dot{u}_{h}\right\|_{L^{3 / 2}(\Omega)}$ and $\left\|\operatorname{div} \dot{u}_{h}\right\|_{L^{2}(\Omega)}$ are bounded, we have $\dot{u} \in L^{3 / 2}(\Omega)$, $\operatorname{div} \dot{u} \in L^{2}(\Omega)$ and

$$
\dot{u}_{h} \rightarrow \dot{u} \quad \text { weakly in } L^{3 / 2}(\Omega),
$$$$
\operatorname{div} \dot{u}_{h} \rightarrow \operatorname{div} \dot{u} \quad \text { weakly in } L^{2}(\Omega) \text {. }
$$

In a similar way, we get that $\|\varepsilon(\dot{u})\|_{L^{2}(A)}<+\infty$ and that

$$
\varepsilon\left(\dot{u}_{h}\right) \rightarrow \varepsilon(\dot{u}) \text { weakly in } L^{2}(A) \text {. }
$$

Now we want to prove that

$$
\left[\sigma^{D} \cdot \varepsilon^{D}\left(\dot{u}_{h}\right)\right] \rightarrow\left[\sigma^{D} \cdot \varepsilon^{D}(\dot{u})\right] \text { weakly as measures in } \Omega .
$$

In order to do that, consider the measures $\left.\varepsilon^{D}\left(\dot{u}_{h}\right)\right|_{C}$ defined as

$$
\left.\int_{B} \varepsilon^{D}\left(\dot{u}_{h}\right)\right|_{C}=\int_{B \cap C} \varepsilon^{D}\left(\dot{u}_{h}\right) \text { for all Borel sets } B \subset \Omega
$$


(recall the definition of the sets $C$ and $A$ in Sec. 1) and let us prove that

$$
\left.\left.\int_{\Omega} g \cdot \varepsilon^{D}\left(\dot{u}_{h}\right)\right|_{C} \rightarrow \int_{\Omega} g \cdot \varepsilon^{D}(\dot{u})\right|_{C} \text { for all } g \in C_{0}^{0}\left(\Omega, \mathbb{R}^{9}\right) .
$$

Take a function $g \in C_{0}^{0}\left(\Omega, \mathbb{R}^{9}\right)$, take a number $\delta>0$, and let $\psi \in C_{0}^{0}(\Omega)$ be such that

$$
\begin{gathered}
\psi(x)=1 \quad \text { for all } x \in C, \\
\int_{A}|\psi g|^{2}<\delta^{2} .
\end{gathered}
$$

Then we have

$$
\left.\int_{\Omega} g \cdot \varepsilon^{D}(\dot{u})\right|_{C}=\int_{C}(\psi g) \cdot \varepsilon^{D}(\dot{u})
$$

(and similarly for all $\dot{u}_{h}$ ) so that

$$
\begin{aligned}
& \left|\int_{\Omega} g \cdot \varepsilon^{D}\left(\dot{u}_{h}\right)\right|_{C}-\left.\int_{\Omega} g \cdot \varepsilon^{D}(\dot{u})\right|_{C} \mid \\
& \quad \leqslant\left|\int_{\Omega}(\psi g) \cdot \varepsilon^{D}\left(\dot{u}_{h}\right)-\int_{\Omega}(\psi g) \cdot \varepsilon^{D}(\dot{u})\right|+\left|\int_{A}(\psi g) \cdot \varepsilon^{D}\left(\dot{u}_{h}\right)-\int_{A}(\psi g) \cdot \varepsilon^{D}(\dot{u})\right|
\end{aligned}
$$

where the first term on the right side of (4.6) goes to zero for $h \rightarrow \infty$ and the last term is less than

$$
\delta \cdot\left(\sup _{h}\left\|\varepsilon^{D}\left(\dot{u}_{h}\right)\right\|_{L^{2}(A)}+\left\|\varepsilon^{D}(\dot{u})\right\|_{L^{2}(A)}\right) \leqslant 2 \delta c .
$$

Taking the limit of both sides in (4.6) for $h \rightarrow \infty$ we get (4.5). Formula (4.4) follows now from (4.5) just by looking up definitions.

By (4.4) we get

$$
\varepsilon^{D}\left(\dot{u}_{h}\right)_{\perp} \rightarrow \varepsilon^{D}(\dot{u})_{\perp} \quad \text { weakly as measures in } \Omega
$$

and, since

$$
\left\|\varepsilon^{D}\left(\dot{u}_{h}\right)_{\perp}\right\|_{L^{2}(\Omega)} \leqslant\left\|\dot{u}_{h}\right\|_{P_{o}(\Omega)} \leqslant c
$$

we also get

$$
\varepsilon^{D}\left(\dot{u}_{h}\right)_{\perp} \rightarrow \varepsilon^{D}(\dot{u})_{\perp} \quad \text { weakly in } L^{2}(\Omega) .
$$

Finally, as we also have

$$
\int_{\Omega}\left[\sigma^{D} \cdot \varepsilon^{D}\left(\dot{u}_{h}\right)\right]^{+} \leqslant \sqrt{2} K c, \quad \int_{\Omega}\left[\sigma^{D} \cdot \varepsilon^{D}\left(\dot{u}_{h}\right)\right]^{-} \leqslant \sqrt{2} K c,
$$

again possibly taking a subsequence, we have

$$
\begin{aligned}
& {\left[\sigma^{D} \cdot \varepsilon^{D}\left(\dot{u}_{h}\right)\right]^{+} \rightarrow \mu_{1}} \\
& {\left[\sigma^{D} \cdot \varepsilon^{D}\left(\dot{u}_{h}\right)\right]^{-} \rightarrow \mu_{2}}
\end{aligned} \quad \text { weakly as measures in } \Omega
$$

where $\mu_{1}$ and $\mu_{2}$ are positive measures of finite mass in $\Omega$, and one can find a non-negative 
function $\gamma(x) \in L^{2}(\Omega)$ such that $\mu_{2}(B)=\int_{B} \gamma(x) d x$ for all Borel subset $B$ of $\Omega$ and

$$
\left[\sigma^{D} \cdot \varepsilon^{D}\left(\dot{u}_{h}\right)\right]^{-} \rightarrow \gamma \text { weakly in } L^{2}(\Omega) \text {. Q.E.D. }
$$

Now we are going to prove a closure theorem.

Theorem 4.3. Let $\sigma$ and $\dot{g}$ be as in Theorem 4.1. Let $\dot{u}_{h} \in U, \dot{u} \in P_{\sigma}(\Omega)$ be such that

$$
\begin{gathered}
\dot{u}_{h} \rightarrow \dot{u} \quad P_{\sigma}(\Omega) \text {-weakly, } \\
\left\|\dot{u}_{h}\right\|_{P_{o}(\Omega)} \leqslant c<+\infty \quad \text { for all } h \in \mathbb{N} ;
\end{gathered}
$$

then $u \in U$, i.e. $u$ satisfies the boundary conditions (4.2).

Proof. Consider the functions $\dot{v}_{h}$ defined by

$$
\dot{v}_{h}= \begin{cases}\dot{u}_{h} & \text { in } \Omega, \\ \dot{g} & \text { in } \Omega_{1} \backslash \bar{\Omega} .\end{cases}
$$

By Theorem 2.3 we know that $\dot{v}_{h} \in P_{\sigma}\left(\Omega_{1}\right)$ for all $h$, and we have

$$
\left\|\dot{v}_{h}\right\|_{P_{o}\left(\Omega_{1}\right)} \leqslant\left\|\dot{u}_{h}\right\|_{P_{o}(\Omega)}+\|\dot{g}\|_{P_{o}\left(\Omega_{1} \backslash \bar{\Omega}\right)}+\int_{\partial \Omega}\left|\varepsilon^{D}\left(\dot{v}_{h}\right)\right|
$$

where

$$
\int_{\partial \Omega}\left|\varepsilon^{D}\left(\dot{v}_{h}\right)\right|=\frac{1}{\sqrt{2}} \int_{\partial \Omega}\left|\dot{u}_{h}-\dot{g}\right| d \mathcal{F}^{2} \leqslant \operatorname{cost}\left(\left\|\dot{u}_{h}\right\|_{\mathrm{BD}(\Omega)}+\|\dot{g}\|_{\mathrm{BD}(\Omega)}\right) .
$$

Now, by Theorem 4.2, there is a subsequence $\dot{v}_{h_{j}}$ that converges $P_{o}\left(\Omega_{1}\right)$-weakly to some function $\dot{v} \in P_{\sigma}\left(\Omega_{1}\right)$ and it must be

$$
\dot{v}= \begin{cases}\dot{u} & \text { in } \Omega \\ \dot{g} & \text { in } \Omega_{1} \backslash \bar{\Omega}\end{cases}
$$

Hence, by Theorem 2.1, we see that $\dot{u}$ satisfies conditions (4.2). Q.E.D.

Last, we give a semicontinuity result.

THEOREM 4.4. The function $E_{1}(\dot{u})$ is sequentially lower-semicontinuous with respect to the $P_{\sigma}(\Omega)$-weak convergence.

Proof. Recall that

$$
\begin{aligned}
E_{1}(\dot{u})= & \frac{K_{0}}{2} \int_{\Omega}(\operatorname{div} \dot{u})^{2} d x+\mu \int_{\Omega}\left|\varepsilon^{D}(\dot{u})_{\perp}\right|^{2} d x \\
& +\mu \int_{\Omega}\left\{\left[\sigma^{D} \cdot \varepsilon^{D}(\dot{u})\right]^{-}(x)\right\}^{2} d x .
\end{aligned}
$$

By well-known theorems, the first two terms on the right side of (4.7) are lower-semicontinuous with respect to the weak convergence in $L^{2}(\Omega)$ of $\operatorname{div} \dot{u}_{h}$ and $\varepsilon^{D}\left(\dot{u}_{h}\right)_{\perp}$. For the same theorems we also have, for any sequence $u_{n} \rightarrow u P_{\sigma}(\Omega)$-weakly:

$$
\min _{h \rightarrow \infty} \lim _{\Omega}\left\{\left[\sigma^{D} \cdot \varepsilon^{D}\left(\dot{u}_{h}\right)\right]^{-}(x)\right\}^{2} d x \geqslant \int_{\Omega}|\gamma(x)|^{2} d x
$$


where, by Remark 4.3,

$$
\int_{\Omega}|\gamma(x)|^{2} d x \geqslant \int_{\Omega}\left\{\left[\sigma^{D} \cdot \varepsilon^{D}(\dot{u})\right]^{-}(x)\right\}^{2} d x \text {. Q.E.D. }
$$

Now we are ready to prove our existence theorem for Problem (P.2).

Theorem 4.5. Given $\sigma$ and $\dot{g}$ as in Problem (P.2), there exists a minimum of the functional $E_{1}(\dot{u})$ among the vector fields $\dot{u} \in P_{\sigma}(\Omega)$ that satisfy the boundary condition (4.2).

Proof. We have

$$
-M \leqslant \inf _{\dot{v} \in U} E_{1}(\dot{v}) \leqslant E_{1}(\dot{g})<+\infty .
$$

Take a sequence $\dot{u}_{h} \in U$ such that

$$
\lim _{h \rightarrow \infty} E_{1}\left(\dot{u}_{h}\right)=\inf _{\dot{v} \in U} E_{1}(\dot{v}) .
$$

We have, by Theorem 4.1,

$$
\left\|\dot{u}_{h}\right\|_{P_{o}(\Omega)} \leqslant E_{1}\left(\dot{u}_{h}\right)+M \leqslant \cos t<+\infty
$$

and by Theorem 4.2 , possibly taking a subsequence, we get

$$
\dot{u}_{h} \rightarrow \dot{u} \quad P_{\sigma}(\Omega) \text {-weakly }
$$

for some $\dot{u} \in P_{\sigma}(\Omega)$. By Theorem 4.3 we know that $\dot{u} \in U$ and by Theorem 4.4 we obtain

$$
E_{1}(\dot{u})=\inf _{\dot{v} \in U} E_{1}(\dot{v}) \text {. Q.E.D. }
$$

Obviously, one also has the following result.

THEOREM 4.6. Under the conditions of Theorem 4.5 , if $\|\dot{f}\|_{L^{3}(\Omega)}$ is sufficiently small, there exists in $V$ a minimum point for the functional

$$
E_{1}(\dot{u})-\int_{\Omega} \dot{f} \dot{u} d x
$$

5. In this section we shall obtain a weak solution to Problem (P.1) (in the case that $\Gamma_{D}=\partial \Omega$ ) from every solution to Problem (P.2).

Let $\dot{u}$ be a solution to Problem (P.2) and set

$$
\begin{aligned}
\dot{\sigma}^{D} & =2 \mu e^{D}(\dot{u}), \\
\operatorname{trace} \dot{\sigma} & =3 K_{0} \operatorname{div} \dot{u}, \\
\lambda & =\frac{1}{2 K^{2}}\left[\sigma^{D} \cdot \varepsilon^{D}(\dot{u})\right]^{+} .
\end{aligned}
$$

It is clear that the constitutive laws for the rates are satisfied in the sense that

$$
\varepsilon(\dot{u})=\frac{1}{9 K_{0}} \operatorname{trace} \dot{\sigma} I+\frac{1}{2 \mu} \dot{\sigma}^{D}+\lambda \sigma^{D}
$$

where $\lambda$ is a positive measure which is zero in the set $\left\{x \in \Omega|| \sigma^{D}(x) \mid<\sqrt{2} K\right\}$ and is supported by the set $C=\left\{x \in \Omega|| \sigma^{D}(x) \mid=\sqrt{2} K\right\}$; moreover, in the set $C, \lambda$ is the 
positive part of the measure $\sigma^{D} \cdot \varepsilon^{D}(\dot{u})$; hence $\lambda$ is concentrated only in the set where $\sigma^{D} \cdot \varepsilon^{D}(\dot{u}) \geqslant 0$, as was required in (0.4). Obviously we have also

$$
\dot{\sigma}_{i j}^{D} \cdot \sigma_{i j}^{D} \leqslant 0
$$

in the set $C$, and we remark that $\dot{\sigma}_{i j}^{D} \cdot \sigma_{i j}^{D}=0$ where $\lambda>0$.

Actually, $\dot{\sigma}^{D}$ is not necessarily defined for all $x \in \Omega$ and (5.2) makes sense only almost everywhere in $\Omega$, but we can redefine $\dot{\sigma}^{D}$ in a set of Lebesgue measure zero in order to have (5.2) true for all $x \in C$.

To show that $(\dot{u}, \dot{\sigma}, \lambda)$ is indeed a solution to Problem (P.1), we still have to check the equilibrium condition (0.1) (we do not have (0.2) since $\Gamma_{N}$ is empty). To do that we shall use the Euler equation of the functional $E_{1}(\dot{u})$ :

$$
\left.\frac{d}{d t} E_{1}(\dot{u}+t \dot{\varphi})\right|_{t=0}=0
$$

for all $\dot{\varphi} \in P_{\sigma}(\Omega)$ such that

$$
\begin{aligned}
& \dot{\varphi}=0 \quad \mathcal{H}^{2} \text {-a.e. in }\left\{x \in \partial \Omega|| L_{t}(x) \mid<K\right\}, \\
& \dot{\varphi} \cdot n=0, \quad[\dot{\varphi} \cdot L]^{-}=0, \quad \tau^{D}(\dot{\varphi})-[\dot{\varphi} \cdot L] \sigma^{D}=0 \quad \mathcal{H}^{2} \text {-a.e. in } \partial \Omega .
\end{aligned}
$$

We shall only need to consider (5.3) in the case that $\dot{\varphi}$ is also in $C^{1}(\bar{\Omega})$.

We have

$$
\begin{aligned}
\left.\frac{d}{d t} E_{1}(\dot{u}+t \dot{\varphi})\right|_{t=0}= & K_{0} \int_{\Omega} \operatorname{div} \dot{u} \operatorname{div} \dot{\varphi} d x+2 \mu \int_{\Omega} \varepsilon^{D}(\dot{u})_{\perp} \varepsilon^{D}(\dot{\varphi}) d x \\
& +\left.\mu \frac{d}{d t} \int_{\Omega}\left\{\left[\sigma^{D} \cdot\left(\varepsilon^{D}(\dot{u})+t \varepsilon^{D}(\dot{\varphi})\right)\right]^{-}(x)\right\}^{2} d x\right|_{t=0}
\end{aligned}
$$

where the last term is equal to

$$
\begin{gathered}
\left.2 \mu \int_{\Omega}\left[\sigma^{D} \cdot \varepsilon^{D}(\dot{u})\right]^{-}(x) \frac{d}{d t}\left\{\left[\sigma^{D} \cdot\left(\varepsilon^{D}(\dot{u})+t \varepsilon^{D}(\dot{\varphi})\right)\right]^{-}(x)\right\}\right|_{t=0} d x \\
=-2 \mu \int_{\Omega}\left[\sigma^{D} \cdot \varepsilon^{D}(\dot{u})\right]^{-}(x)\left(\sigma^{D} \cdot \varepsilon^{D}(\dot{\varphi})\right) d x
\end{gathered}
$$

because, if $\left[\sigma^{D} \cdot \varepsilon^{D}(\dot{u})\right]^{-}(x)>0$, we have, for all $t$ in a neighborhood of zero,

$$
\left[\sigma^{D} \cdot \varepsilon^{D}(\dot{u})+t \sigma^{D} \cdot \varepsilon^{D}(\dot{\varphi})\right]^{-}(x)=\left[\sigma^{D} \cdot \varepsilon^{D}(\dot{u})\right]^{-}(x)-t\left(\sigma^{D} \cdot \varepsilon^{D}(\dot{\varphi})\right)
$$

while, if $\left[\sigma^{D} \cdot \varepsilon^{D}(\dot{u})\right]^{-}(x)=0$, we are not interested in the value of the derivative in (5.4), as it is multiplied by zero.

Concluding, we have that the Euler equation for the functional $E_{1}(\dot{u})$ is, for all admissible $\dot{\varphi}$ in $C^{1}(\bar{\Omega})$,

$$
K_{0} \int_{\Omega} \operatorname{div} \dot{u} \operatorname{div} \dot{\varphi}+2 \mu \int_{\Omega} \varepsilon^{D}(\dot{u})_{\perp} \varepsilon^{D}(\varphi)-2 \mu \int_{\Omega}\left[\sigma^{D} \cdot \varepsilon^{D}(\dot{u})\right]^{-} \frac{\sigma^{D}}{2 K^{2}} \cdot \varepsilon^{D}(\dot{\varphi})=0
$$

and that we can also write

$$
\int_{\Omega}\left(K_{0} \operatorname{div} \dot{u}+2 \mu e^{D}(\dot{u})\right) \varepsilon(\dot{\varphi})=\int_{\Omega} \dot{\sigma} \cdot \varepsilon(\dot{\varphi})=0 .
$$


Now, as (5.5) holds in particular for $\dot{\varphi} \in C_{0}^{\infty}(\Omega)$ we have

$$
\dot{\sigma}_{i j, j}=0 \text { in the sense of distributions in } \Omega
$$

which is the equilibrium condition (0.1) in our case, i.e. with $\dot{f}=0$. Obviously, if we had taken a minimum $\dot{u}$ of the functional

$$
E_{1}(\dot{u})-\int_{\Omega} \dot{f} \dot{u} d x
$$

we would have obtained in the same way that $\dot{\sigma}_{i j \cdot j}+\dot{f}_{i}=0$ in the sense of distributions in $\Omega$.

6. In this section we shall consider Problem (P.1) in the case $\Gamma_{N}=\partial \Omega, \Gamma_{D}=\varnothing$, i.e. in the case of Neumann boundary conditions. As we did for the Dirichlet problem, first we shall find a rate of displacement $\dot{u}$ that minimizes a suitable energy functional $E_{2}(\dot{u})$, then we shall obtain from $\dot{u}$ a weak solution to Problem (P.1).

We assume that

$$
\dot{f} \in L^{3}(\Omega), \quad \dot{F} \in L^{\infty}(\Omega) ;
$$

we assume also that $\sigma \in \Sigma(\Omega)$, with $\sigma^{D} \in C^{0}(\Omega)$, is a given stress state satisfying the safe load condition (H.4.1), and we set

$$
E_{2}(\dot{u})=\frac{K_{0}}{2} \int_{\Omega}(\operatorname{div} \dot{u})^{2} d x+\mu \int_{\Omega}\left|e^{D}(\dot{u})\right|^{2} d x-\int_{\Omega} \dot{f} \dot{u} d x-\int_{\partial \Omega} \dot{F} \dot{u} d \mathcal{H}^{2}
$$

Define a linear functional $T_{0}: \operatorname{BD}(\Omega) \rightarrow g=\left\{a \wedge x+b \mid a, b \in \mathbb{R}^{3}\right\}$ as follows [5]: take a fixed point $x_{0} \in \Omega$ and a positive number $R<\operatorname{dist}\left(x_{0}, \partial \Omega\right)$, then set

$$
\left[\left(T_{0} u\right)(x)\right]_{j}=\frac{1}{2} \sum_{i=1}^{3}\left(\rho_{i j}(u)-\rho_{j i}(u)\right)\left(x-x_{0}\right)_{i}+\eta_{j}(u)
$$

where

$$
\begin{aligned}
\eta_{i}(u) & =\frac{3}{4 \pi R^{3}} \int_{B_{R}\left(x_{0}\right)} u_{i}(y) d y, \\
\rho_{i j}(u) & =\frac{4}{\pi R^{4}} \int_{\left\{y \in B_{R}\left(x_{0}\right) \mid\left(y-x_{0}\right) \cdot \theta_{j}>0\right\}}\left(u_{i}(y)-\eta_{i}(u)\right) d y
\end{aligned}
$$

and $\left\{\boldsymbol{\theta}_{1}, \boldsymbol{\theta}_{2}, \boldsymbol{\theta}_{3}\right\}$ is an orthonormal basis of $\mathbb{R}^{3}$.

Notice that $\left\|T_{0} u\right\|_{\mathbf{R}^{3} \times \mathbf{R}^{3}} \leqslant C\|u\|_{L^{\prime}(\Omega)}$.

Now consider the following

Problem (P.3). Minimize the functional $E_{2}(\dot{u})$ among the functions $\dot{u} \in P_{\sigma}(\Omega)$ such that $T_{0}(\dot{u})=0$.

In order to find a solution to Problem (P.3), we have to prove the coerciveness and the semicontinuity of $E_{2}(\dot{u})$ and, to do that, we have to impose some requirements on $\dot{f}, \dot{F}$. We shall give a sufficient and almost necessary condition on $\dot{f}, \dot{F}$ in order for Problem (P.3) to have a solution.

Let us begin with a few definitions. 
Definition 6.1. We shall say that a Borel function $\dot{\alpha}: \Omega \rightarrow \mathbb{R}^{9}, \dot{\alpha}=\left\{\dot{\alpha}_{i j}\right\}$ belongs to the space $\Sigma_{\sigma}(\Omega)$ if

$$
\begin{gathered}
\dot{\alpha}_{i j}(x)=\dot{\alpha}_{j i}(x) \quad \text { for all } x \in \Omega, \\
\int_{\Omega}|\dot{\alpha}(x)|^{2} d x<+\infty, \\
\dot{\alpha}^{D}(x) \cdot \sigma^{D}(x) \leqslant 0 \quad \text { for all } x \in \Omega \text { such that }\left|\sigma^{D}(x)\right|=\sqrt{2} K .
\end{gathered}
$$

Definition 6.2. We define a function

$$
\langle\dot{\alpha}, \dot{\varphi}\rangle_{\Omega}^{1}: \Sigma_{\sigma}(\Omega) \times P_{\sigma}(\Omega) \rightarrow[-\infty,+\infty)
$$

as

$$
\begin{aligned}
\langle\dot{\alpha}, \dot{\varphi}\rangle_{\Omega}^{1}= & \frac{1}{3} \int_{\Omega} \operatorname{trace} \dot{\alpha} \operatorname{div} \dot{\varphi} d x+\int_{\Omega} \dot{\alpha} \cdot e^{D}(\dot{\varphi}) d x \\
& +\int_{\Omega} \alpha^{D} \cdot\left[\sigma^{D} \cdot \varepsilon^{D}(\dot{\varphi})\right]^{+} \sigma^{D}
\end{aligned}
$$

where

$$
\int_{\Omega} \alpha^{D} \cdot\left[\sigma^{D} \cdot \varepsilon^{D}(\dot{\varphi})\right]^{+} \sigma^{D}=\int_{\Omega}\left(\alpha^{D} \cdot \sigma^{D}\right)\left[\sigma^{D} \cdot \varepsilon^{D}(\dot{\varphi})\right]^{+}
$$

is defined as the integral with respect to the positive Radon measure $\left[\sigma^{D} \cdot \varepsilon^{D}(\dot{\varphi})\right]^{+}$of the Borel function $\left(\alpha^{D} \cdot \sigma^{D}\right)$, which is non-positive on the support of $\left[\sigma^{D} \cdot \varepsilon^{D}(\dot{\varphi})\right]^{+}$.

Definition 6.3. We shall say that $\dot{\alpha} \in \Sigma_{o}(\Omega)$ is $(\dot{f}, \dot{F})$-admissible if

$$
\langle\dot{\alpha}, \dot{\varphi}\rangle_{\Omega}=\int_{\Omega} \dot{f} \dot{\varphi}+\int_{\partial \Omega} \dot{F} \dot{\varphi} \quad \text { for all } \dot{\varphi} \in P_{\sigma}(\Omega) .
$$

Notice that (6.3) is just a weak formulation of

$$
\begin{aligned}
\dot{\alpha}_{i j, j}+\dot{f}_{i}=0 & \text { in } \Omega, \\
\dot{\alpha}_{i j} n_{j}=\dot{F}_{i} & \text { on } \partial \Omega .
\end{aligned}
$$

If there exists a $(\dot{f}, \dot{F})$-admissible stress rate $\dot{\alpha}$, then we can estimate the force terms in the functional $E_{2}(\dot{u})$.

LEMMA 6.1. If $\dot{\alpha} \in \Sigma_{\sigma}(\Omega)$ is $(\dot{f}, \dot{F})$-admissible, we have, for all $\dot{u} \in P_{\sigma}(\Omega)$,

$$
\begin{aligned}
\frac{1}{3}\|\operatorname{trace} \dot{\alpha}\|_{L^{2}}\|\operatorname{div} \dot{u}\|_{L^{2}}+\left\|\dot{\alpha}^{D}\right\|_{L^{2}}\left\|e^{D}(\dot{u})\right\|_{L^{2}}-\frac{1}{2 K^{2}} \int_{\Omega}\left(\dot{\alpha}^{D} \cdot \sigma^{D}\right)\left[\sigma^{D} \cdot \varepsilon^{D}(\dot{u})\right]^{+} \\
\geqslant-\int_{\Omega} \dot{f} \dot{u}-\int_{\partial \Omega} \dot{F} \dot{u} \geqslant-\frac{1}{3}\|\operatorname{trace} \dot{\alpha}\|_{L^{2}}\|\operatorname{div} \dot{u}\|_{L^{2}}-\left\|\dot{\alpha}^{D}\right\|_{L^{2}}\left\|e^{D}(\dot{u})\right\|_{L^{2}} .
\end{aligned}
$$

Proof. Obvious.

Now we have a coercivity result for $E_{2}(\dot{u})$.

Theorem 6.1. If there exists a $(\dot{f}, \dot{F})$-admissible stress rate field $\dot{\alpha}$ then the functional $E_{2}(\dot{u})$ is coercive on $P_{\sigma}(\Omega) \cap\left\{T_{0} \dot{u}=0\right\}$. More precisely, there exist two positive numbers 
$c_{0}, M$ (depending on $\mu, K, K_{0}, \sigma, \dot{\alpha}, \Omega$ but not on $\dot{u}$ ) such that

$$
E_{2}(\dot{u}) \geqslant c_{0}\|\dot{u}\|_{P_{\sigma}(\Omega)}-M \text { for all } \dot{u} \in P_{\sigma}(\Omega) \text { with } T_{0} \dot{u}=0 .
$$

Proof. By Theorem 3.3 we have

$$
-\langle\sigma, \dot{u}\rangle_{\Omega}+\int_{\Omega} \sigma^{D} \cdot \varepsilon^{D}(\dot{u})+\frac{1}{3} \operatorname{trace} \sigma \operatorname{div} \dot{u}=0 .
$$

Adding (6.5) to $E_{2}(\dot{u})$, arguing as in the proof of Theorem 4.1, and taking into account Lemma 6.1 we get

$$
E_{2}(\dot{u}) \geqslant c_{0}^{1}\left\{\int_{\Omega}(\operatorname{div} \dot{u})^{2} d x+\int_{\Omega}\left|e^{D}(\dot{u})\right|^{2} d x+\int_{\Omega}|\varepsilon(\dot{u})|\right\}-M^{1}
$$

and, recalling Theorem 1.1, as $T_{0} \dot{u}=0$, we get (6.4). Q.E.D.

Remark 6.1. Notice that we didn't have to assume that $\sigma$ is defined in an open set $\Omega_{1} \supset \supset \Omega$.

Remark 6.2. It is also possible to prove (6.4) under the condition that $\|\dot{f}\|_{L^{3}},\|\dot{F}\|_{L^{\infty}}$ are sufficiently small.

We have already proved (see Theorem 4.4) the lower-semicontinuity of the first two terms of the functional $E_{2}(\dot{u})$ and we only need to see what happens for the force terms.

THEOREM 6.2. If there exists a $(\dot{f}, \dot{F})$-admissible stress rate field $\dot{\alpha}$ such that

$$
\dot{\alpha}^{D}(x) \cdot \sigma^{D}(x)=0 \text { for all } x \in \Omega \text { with }\left|\sigma^{D}(x)\right|=\sqrt{2} K
$$

then the functional $E_{2}(\dot{u})$ is sequentially lower semicontinuous with respect to the $P_{\sigma}(\Omega)$-weak convergence.

Proof. Take $\dot{u}_{h}, \dot{u} \in P_{\sigma}(\Omega)$ such that $\dot{u}_{h} \rightarrow \dot{u} P_{\sigma}(\Omega)$-weakly. By (6.1), (6.3) and (6.6) we have, for all $h \in \mathbb{N}$,

$$
\begin{aligned}
-\int_{\Omega} \dot{f} \dot{u}_{h}-\int_{\partial \Omega} \dot{F} \dot{u}_{h}= & -\frac{1}{3} \int_{\Omega} \operatorname{trace} \dot{\alpha} \operatorname{div} \dot{u}_{h}-\int_{\Omega} \dot{\alpha}^{D} \cdot \varepsilon^{D}\left(\dot{u}_{h}\right)_{\perp} \\
& +\frac{1}{2 K^{2}} \int_{\Omega} \dot{\alpha}^{D} \cdot\left[\sigma^{D} \cdot \varepsilon^{D}\left(\dot{u}_{h}\right)\right] \sigma^{D}
\end{aligned}
$$

where the last term is zero because $\dot{\alpha}^{D} \cdot \sigma^{D}$ is zero in the support of $\left[\sigma^{D} \cdot \varepsilon^{D}\left(\dot{u}_{h}\right)\right]$. Now, taking the limit for $h \rightarrow \infty$, we get

$$
\begin{aligned}
\lim _{h \rightarrow \infty}\left\{-\int_{\Omega} \dot{f}_{h}-\int_{\Omega} \dot{F}_{h} \dot{u}_{h}\right\} & \geqslant-\frac{1}{3} \int_{\Omega} \operatorname{trace} \dot{\alpha} \operatorname{div} \dot{u}-\int_{\Omega} \alpha^{D} \cdot \varepsilon^{D}(\dot{u})_{\perp} \\
& =-\langle\dot{\alpha}, \dot{u}\rangle_{\Omega}=-\int_{\Omega} \dot{f} \dot{u}-\int_{\partial \Omega} \dot{F} \dot{u}
\end{aligned}
$$

and, taking into account also Theorem 4.4, our theorem is proved. Q.E.D.

Here is the existence theorem for Problem (P.3).

THEOREM 6.3. If there exists a $(\dot{f}, \dot{F})$-admissible stress rate $\dot{\alpha}$ such that (6.6) holds (and under the hypotheses made on $\dot{f}, \dot{F}, \sigma$ at the beginning of this section), then there exists a 
minimum point for the functional $E_{2}(\dot{u})$ among the functions $\dot{u} \in P_{\sigma}(\Omega)$ such that $T_{0} \dot{u}=0$.

Proof. Notice that the functional $E_{2}$ is not identically $+\infty$. Let $\dot{u}_{h}$ be a minimizing sequence. Then: $\dot{u}_{h}$ is bounded in $P_{\sigma}(\Omega)$ by Theorem 6.1 ; there is a subsequence $\dot{u}_{h_{j}}$ that converges $P_{\sigma}(\Omega)$-weakly to some $\dot{u} \in P_{\sigma}(\Omega)$ by Theorem 4.2 ; as $T_{0} \dot{u}_{h}=0$ for all $h$ we also have $T_{0} \dot{u}=0$, and $\dot{u}$ is a minimum point for $E_{2}$ by Theorem 6.2. Q.E.D.

For any solution $\dot{u}$ to Problem (P.3), define $\dot{\sigma}$ and $\lambda$ as in (5.1). Again, we have that the constitutive laws for the rates are satisfied by definition, and we have to see what happens for the equilibrium conditions. In this case, we have that the Euler equation (see Sec. 5) is

$$
\int_{\Omega}\left(K_{0} \operatorname{div} \dot{u} I+2 \mu e^{D}(\dot{u})\right) \varepsilon(\dot{\varphi})-\int_{\Omega} \dot{f} \dot{\varphi}-\int_{\partial \Omega} \dot{F} \dot{\varphi}=0
$$

for all $\dot{\varphi} \in P_{\sigma}(\Omega)$ such that $T_{0} \dot{\varphi}=0$; hence we have

$$
\int_{\Omega} \dot{\sigma}_{i j} \varepsilon_{i j}(\dot{\varphi})-\int_{\Omega} \dot{f}\left[\dot{\varphi}-T_{0} \dot{\varphi}\right]-\int_{\partial \Omega} \dot{F}\left[\dot{\varphi}-T_{0} \dot{\varphi}\right]=0
$$

for all $\dot{\varphi} \in P_{\sigma}(\Omega)$, where by (6.3) and (6.1)

$$
\int_{\Omega} \dot{f} \cdot T_{0} \dot{\varphi}+\int_{\partial \Omega} \dot{F} \cdot T_{0} \dot{\varphi}=\left\langle\dot{\alpha}, T_{0} \dot{\varphi}\right\rangle_{\Omega}^{1}=0
$$

as $\varepsilon\left(T_{0} \dot{\varphi}\right)=0$. In conclusion, we have that (6.7) holds for all $\dot{\varphi} \in P_{\sigma}(\Omega)$ and, taking $\dot{\varphi} \in C_{0}^{\infty}(\Omega)$, we have that

$$
\dot{\boldsymbol{\sigma}}_{i j, j}+\dot{f}_{i}=0
$$

in $\Omega$ in the sense of distributions, while formula (6.7) for $\dot{\varphi} \in C^{\infty}(\bar{\Omega})$ is a weak formulation of

$$
\dot{\boldsymbol{\sigma}}_{i j} n_{j}=\dot{F}_{i} \quad \text { on } \partial \Omega \text {. }
$$

Remark 6.3. Obviously, $\dot{\sigma}$ is a $(\dot{f}, \dot{F})$-admissible stress rate field; hence a necessary condition to have a solution to Problem (P.3) is that there exist a $(\dot{f}, \dot{F})$-admissible stress rate field. We have seen that this condition is also sufficient, if we also assume (6.6). It would be nicer if one could prove the existence theorem without using (6.6). It should be noted, however, that condition (6.6) also is a natural one, in fact, this condition is necessary on $(d f / d t)(t),(d F / d t)(t)$ at each instant of time, if one wants to have a solution $\{u(t), \sigma(t)\}$ to the quasi-static problem such that $\sigma(t)$ is a differentiable function of $t$.

We end this section by showing that the stress rate field $\dot{\sigma}$ minimizes a suitable complementary energy functional, among the $(\dot{f}, \dot{F})$-admissible stress rate fields.

THEOREM 6.4. Let $\dot{u}$ be a solution to problem (P.3), let $\dot{\sigma}$ be defined as in (5.1) and set

$$
\Psi(\dot{\alpha})=\frac{1}{9 K_{0}}(\operatorname{trace} \dot{\alpha})^{2}+\frac{1}{2 \mu}\left|\dot{\alpha}^{D}\right|^{2}
$$


then we have

$$
\int_{\Omega} \Psi(\dot{\sigma}) \leqslant \int_{\Omega} \Psi(\dot{\alpha})
$$

for all $(\dot{f}, \dot{F})$-admissible stress rate fields $\dot{\alpha}$.

Proof. By the convexity of the function $\Psi$ and by (5.1), we get

$$
\int_{\Omega} \Psi(\dot{\alpha})-\int_{\Omega} \Psi(\dot{\sigma}) \geqslant 2 \int_{\Omega}\left(\varepsilon(\dot{u})-\lambda \sigma^{D}\right)(\dot{\alpha}-\dot{\sigma})
$$

and the theorem follows by recalling that we have

$$
\int_{\Omega} \varepsilon(\dot{u}) \dot{\alpha}=\int_{\Omega} \varepsilon(\dot{u}) \dot{\sigma}
$$

because both $\dot{\alpha}$ and $\dot{\sigma}$ are $(\dot{f}, \dot{F})$-admissible; we have

$$
\int_{\Omega}\left(\dot{\sigma} \cdot \sigma^{D}\right)\left[\sigma^{D} \cdot \varepsilon^{D}(\dot{u})\right]^{+}=0
$$

because $\dot{\sigma} \cdot \sigma^{D}=0$ where $\left[\sigma^{D} \cdot \varepsilon^{D}(\dot{u})\right]^{+}$is non-zero, and we have

$$
-\int_{\Omega}\left(\dot{\alpha} \cdot \sigma^{D}\right)\left[\sigma^{D} \cdot \varepsilon^{D}(\dot{u})\right]^{+} \geqslant 0
$$

because $\sigma^{D} \cdot \dot{\alpha} \leqslant 0$ where $\left|\sigma^{D}(x)\right|=\sqrt{2} K$. Q.E.D.

\section{REFERENCES}

[1] G. Anzellotti and M. Giaquinta, Existence of the displacements field for an elasto-plastic body subject to Hencky's law and Von Mises yield condition, Manuscripta Math. 32, 101-136 (1980)

[2] G. Anzellotti and M. Giaquinta, On the existence of the fields of stresses and displacements for an elasto-perfectly plastic body in static equilibrium, 61 (1982): Journal de Mathematiques pures et appliquees

[3] M. E. Bogovski, Solution to the first boundary value problem for the equation of continuity of an incompressible medium, Soviet Math. Dokl. 20, 1094-1098 (1979)

[4] G. Duvaut and J. L. Lions, Les inequations en mécanique et en physique, Dunod, Paris (1972)

[5] R. Kohn, Ph.D. Thesis, Princeton University (1979)

[6] R. Kohn and R. Temam, to appear

[7] W. T. Koiter, General theorems for elasto-plastic solids, in Progress in solid mechanics, 1, edited by J. N. Sneddon and R. Hill, North Holland, Amsterdam (1960)

[8] H. Matthies, G. Strang and E. Christiansen, The saddle point of a differential program, in Energy methods in finite element analysis, edited by Glowinski, Rodin and Zienkiewicz, John Wiley and Sons (1979)

[9] G. Strang and R. Temam, Functions of bounded deformation, Arch. Rat. Mech. Anal. 75, 7-21 (1980)

[10] P. M. Suquet. evolution problems for a class of dissipative materials, Q. Appl. Math. 38, 391-414 (1981)

[11] R. Temam, An existence theorem for a variational problem of plasticity, Proceedings of the Journées P. de Fermat; Atteia, Bancel, editors, Pitman (1980) 